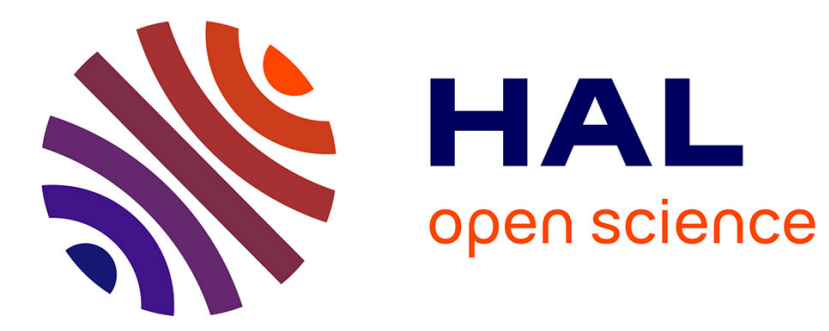

\title{
Shear-stress fluctuations and relaxation in polymer glasses
}

\author{
Ivan Kriuchevskyi, Joachim P Wittmer, Hendrik Meyer, Olivier Benzerara, \\ Jörg Baschnagel
}

\section{- To cite this version:}

Ivan Kriuchevskyi, Joachim P Wittmer, Hendrik Meyer, Olivier Benzerara, Jörg Baschnagel. Shearstress fluctuations and relaxation in polymer glasses. Physical Review E , 2018, 97 (1), pp.012502. 10.1103/PhysRevE.97.012502 . hal-01872457

\section{HAL Id: hal-01872457 https://hal.science/hal-01872457}

Submitted on 20 Sep 2018

HAL is a multi-disciplinary open access archive for the deposit and dissemination of scientific research documents, whether they are published or not. The documents may come from teaching and research institutions in France or abroad, or from public or private research centers.
L'archive ouverte pluridisciplinaire HAL, est destinée au dépôt et à la diffusion de documents scientifiques de niveau recherche, publiés ou non, émanant des établissements d'enseignement et de recherche français ou étrangers, des laboratoires publics ou privés. 


\title{
Shear-stress fluctuations and relaxation in polymer glasses
}

\author{
I. Kriuchevskyi, ${ }^{1,2}$ J. P. Wittmer, ${ }^{1, *}$ H. Meyer, ${ }^{1}$ O. Benzerara, ${ }^{1}$ and J. Baschnagel ${ }^{1}$ \\ ${ }^{1}$ Institut Charles Sadron, Université de Strasbourg \& CNRS, 23 rue du Loess, 67034 Strasbourg Cedex, France \\ ${ }^{2}$ LAMCOS, INSA, 27 av. Jean Capelle, 69621 Villeurbanne Cedex, France
}

(Received 31 October 2017; published 11 January 2018)

\begin{abstract}
We investigate by means of molecular dynamics simulation a coarse-grained polymer glass model focusing on (quasistatic and dynamical) shear-stress fluctuations as a function of temperature $T$ and sampling time $\Delta t$. The linear response is characterized using (ensemble-averaged) expectation values of the contributions (time averaged for each shear plane) to the stress-fluctuation relation $\mu_{\mathrm{sf}}$ for the shear modulus and the shear-stress relaxation modulus $G(t)$. Using 100 independent configurations, we pay attention to the respective standard deviations. While the ensemble-averaged modulus $\mu_{\mathrm{sf}}(T)$ decreases continuously with increasing $T$ for all $\Delta t$ sampled, its standard deviation $\delta \mu_{\mathrm{sf}}(T)$ is nonmonotonic with a striking peak at the glass transition. The question of whether the shear modulus is continuous or has a jump singularity at the glass transition is thus ill posed. Confirming the effective time-translational invariance of our systems, the $\Delta t$ dependence of $\mu_{\text {sf }}$ and related quantities can be understood using a weighted integral over $G(t)$.
\end{abstract}

DOI: 10.1103/PhysRevE.97.012502

\section{INTRODUCTION}

Motivation. The equilibrium shear modulus $\mu_{\mathrm{eq}}(T)$ of crystalline solids is known to vanish discontinuously at the melting point with increasing temperature $T[1,2]$. A natural question which arises is that of the behavior of $\mu_{\mathrm{eq}}(T)$ for amorphous solids and glasses in the vicinity of the glass transition temperature $T_{\mathrm{g}}$. (We assume here that a thermodynamically properly defined static shear modulus does exist. This is not obvious as discussed below.) Two qualitatively different theoretical predictions have been put forward suggesting either a discontinuous jump at the glass transition [3-8] or a continuous (cusplike) transition $[1,2,9-12]$. The predicted jump singularity is a result of mean-field theories $[3,8,13]$ which find the energy barriers for structural relaxation to diverge at $T_{\mathrm{g}}$ so that liquidlike flow stops. However, in experimental or simulated glass formers, the barriers do not diverge abruptly. Such non-mean-field effects are expected to smear out the sharp transition [8]. Another line of recent research has focused on the elastic properties deep in the glass [14-16]. At $T \ll T_{\mathrm{g}}$, a transition in the solid is found, where multiple particle arrangements occur as different competing glassy states. This so-called "Gardner transition" is accompanied by strong fluctuations of $\mu_{\mathrm{eq}}$ (and of higher order elastic moduli) from one glass state to the other $[15,16]$. Interestingly, strong fluctuations of the shear modulus were also observed in self-assembled networks [17] which is a model for vitrimers $[18,19]$. The results of [15-17] beg the question of whether also the glass transition is accompanied by strong fluctuations of shear stresses and moduli.

Our approach. Corroborating a brief account given in Ref. [12], we present here numerical data obtained by means of large-scale molecular dynamics (MD) simulation [20,21] of a standard coarse-grained bead-spring model. This model has already been used in earlier work on the polymer glass

\footnotetext{
*joachim.wittmer@ics-cnrs.unistra.fr
}

transition [12,22-24]. We characterize the shear rigidity in the canonical ensemble [20]:

(i) following the pioneering work by Barrat et al. [1] using as main diagnostics the well-known stress-fluctuation formula $\mu_{\mathrm{sf}}$ for the shear modulus $[1,2,11,12,16,17,22,24-32]$ and its various contributions as defined below in Sec. II;

(ii) by direct computation of the shear-stress relaxation modulus $G(t)$ using the general fluctuation-dissipation relation appropriate for solidlike systems with strong quenched shear stresses [12,24].

Particular attention will be paid to the standard deviations and cross correlations of the different contributions of the two main observables $\mu_{\mathrm{sf}}$ and $G(t)$. We will characterize in detail the (ensemble-averaged) effects of the time preaveraging performed over a finite sampling time $\Delta t$ for each independent configuration and shear plane. This is of importance since the difference between time and ensemble averages corresponds to the standard experimental procedure (properties are first averaged for each shear plane and only then ensemble averaged) and since the detailed averaging procedure matters for all observables characterizing fluctuations [11,17,32].

Key results. We remind [11,29-32] that if a proper sampling time-independent thermodynamic equilibrium modulus $\mu_{\mathrm{eq}}$ characterizing the glass transition existed, this would imply $\mu_{\mathrm{sf}}(\Delta t) \rightarrow \mu_{\mathrm{eq}}$ for sufficiently large sampling times $\Delta t \gg \tau_{\infty}$ and also $G(t) \rightarrow \mu_{\text {eq }}$ for large times $t \gg \tau_{\infty}$ with $\tau_{\infty}(T)$ being the terminal relaxation time of the system. Our numerical results are in fact qualitatively quite different and much more in line with our recent study on self-assembled transient networks [17]. We highlight three key results demonstrated below:

(I) $\mu_{\mathrm{sf}}(T)$ decreases continuously and monotonically for all temperatures $T$ and sampling times $\Delta t$. Being $\Delta t$ dependent, $\mu_{\mathrm{sf}}$ is not an equilibrium storage modulus. Albeit the crossover of $\mu_{\mathrm{sf}}(T)$ at $T_{\mathrm{g}}$ becomes systematically sharper with increasing $\Delta t$, our data are not consistent with a jump singularity. 
(II) The standard deviation $\delta \mu_{\text {sf }}$ is strongly nonmonotonic with respect to $T$ with a remarkable peak at $T_{\mathrm{g}}$. The transition characterized by $\mu_{\mathrm{sf}}(T)$ is thus masked by very strong fluctuations [33].

(III) We demonstrate that $\mu_{\mathrm{sf}}$ is identical to the weighted moment $\mu(\Delta t)$ over the shear-stress relaxation modulus $G(t)$ defined by

$$
\mu(\Delta t) \equiv \frac{2}{\Delta t^{2}} \int_{0}^{\Delta t} d t(\Delta t-t) G(t)
$$

The observed $\Delta t$ dependence of $\mu_{\mathrm{sf}}$ is thus not due to nonequilibrium ("aging") processes but can be traced back to the finite sampling time (time-averaged) stress fluctuations need to explore the phase space. The historically thermodynamically rooted $\mu_{\mathrm{sf}}$ takes due to Eq. (1) the meaning of a "generalized modulus" also containing information about dissipation processes associated to the plastic reorganization of the particle contact network [34]. It thus follows as a corollary from Eq. (1) that the shear viscosity $\eta_{\infty}(T)$ above the glass transition may be obtained using

$$
\mu_{\mathrm{sf}}(\Delta t) \rightarrow \frac{2 \eta_{\infty}(T)}{\Delta t} \text { for } \Delta t \gg \tau_{\infty}(T)
$$

in agreement with the well-known Helfand-Einstein relation for the shear viscosity [20,35-37]. Due to the inevitable too low precision of $G(t)$ for large times [20], especially for supercooled liquids close to the glass transition, this method is shown to be much more precise than the Green-Kubo relation using the asymptotic behavior of the generalized (dynamic) shear viscosity

$$
\eta(\Delta t) \equiv \int_{t=0}^{\Delta t} d t G(t) \quad \text { with } \eta_{\infty}=\lim _{\Delta t \rightarrow \infty} \eta(\Delta t) .
$$

Moreover, Eq. (1) will allow us to express $G(t), \eta(\Delta t)$, and the related generalized terminal relaxation time $\tau(\Delta t)$ in terms of the numerically better behaved $\mu_{\mathrm{sf}}(\Delta t)$.

Outline. This paper is organized as follows. Our polymer model is defined in Sec. II where we also explain technical details concerning the quench protocol, the time series stored, and the different time and ensemble averages computed. We begin the presentation of our numerical results in Sec. III where we focus on the (ensemble-averaged) expectation values of the stress-fluctuation prediction $\mu_{\mathrm{sf}}$ for the shear modulus and its related contributions. Standard deviations, fluctuations, and cross correlations of the different contributions to $\mu_{\mathrm{sf}}$ are discussed in Sec. IV. We turn then in Sec. V to the shearstress relaxation function $G(t)$ and the associated sampling time dependent moments $\mu(\Delta t)$ and $\eta(\Delta t)$. We demonstrate in Sec. VB that $\mu_{\mathrm{sf}}(\Delta t)$ and $\mu(\Delta t)$ are identical. Various important consequences are discussed in Secs. VC and VD. We show especially that Eq. (2) must hold. The standard deviation $\delta G(t)$ of $G(t)$ is considered in Sec. VE. We verify in Sec. VI that our results are not due to finite-size effects. The paper is summarized in Sec. VII A and an outlook on ongoing work is given in Sec. VII B. Appendix A reminds the connection between canonical affine shear transformations and the instantaneous shear stress $\hat{\tau}$ and the affine shear modulus $\hat{\mu}_{\mathrm{A}}$. Focusing in Appendix B on temperatures above the glass transition we determine the shear viscosity $\eta_{\infty}(T)$ and the terminal relaxation time $\tau_{\infty}(T)$ from the $\Delta t$ dependence of

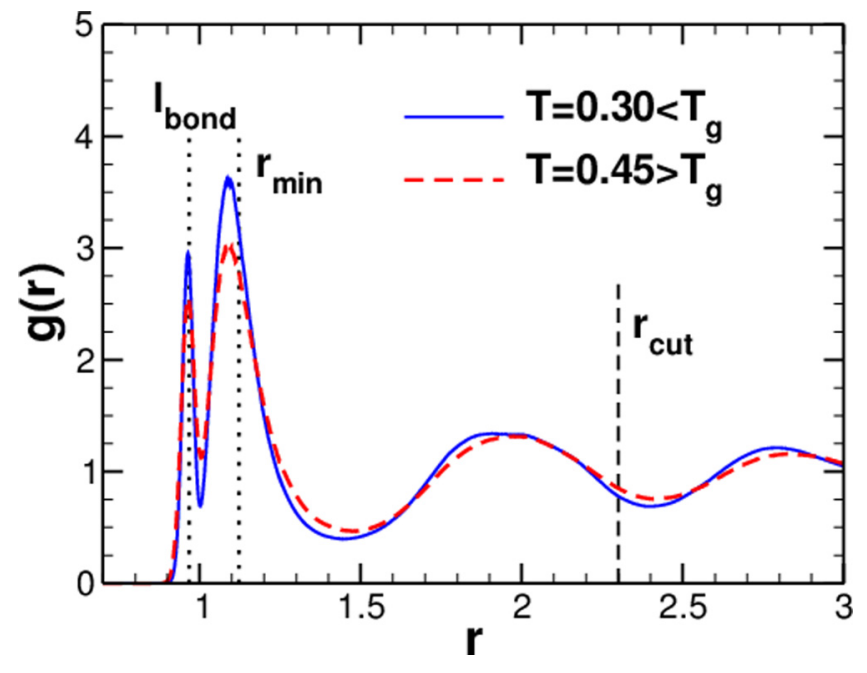

FIG. 1. Radial pair correlation function $g(r)$ for one temperature above and one below the glass transition temperature $T_{\mathrm{g}}$ showing that our coarse-grained polymer model does not crystallize. Also indicated are the equilibrium bond length $l_{\text {bond }}=0.967$, the position of the minimum of the $\mathrm{LJ}$ potential $r_{\min }=2^{1 / 6}$, and the potential cutoff distance $r_{\text {cut }}=2.3$.

$\mu_{\mathrm{sf}}$. Additional details concerning the shear-stress relaxation modulus $G(t)$ are given in Appendix C. The derivation of Eq. (1) for systems with time-translational invariance is given in Appendix D. The generalized terminal relaxation time $\tau(\Delta t)$ is considered in Appendix E.

\section{ALGORITHMICAL DETAILS}

\section{A. Model Hamiltonian}

Our data have been obtained by MD simulation [20] of a bead-spring model already used in earlier work on the polymer glass transition [12,22-24,38]. In this model all monomers, that are not connected by bonds, interact via a monodisperse Lennard-Jones (LJ) potential. LJ units [20] are thus used. To increase the numerical efficiency, the LJ potential is truncated at $r_{\text {cut }}=2.3 \approx 2 r_{\min }$, with $r_{\text {min }}=2^{1 / 6}$ being the potential minimum, and shifted at $r_{\text {cut }}$ to make it continuous. (See Sec. IIF below.) The flexible bonds are represented by a harmonic spring potential $U_{\text {bond }}(r)=\left(k_{\text {bond }} / 2\right)\left(r-l_{\text {bond }}\right)^{2}$ with $r$ being the distance between the beads, $k_{\mathrm{bond}}=1110$ the spring constant, and $l_{\text {bond }}=0.967$ the equilibrium bond length as indicated in Fig. 1.

\section{B. Operational parameters}

As in Refs. [12,24] we focus in this work on data obtained using $m=100$ independent configurations containing $M=$ 3072 chains of length $N=4$. As may be seen from Fig. 1, this chain length is sufficient to avoid the crystallization tendency of the monodisperse LJ beads [23]. It is, however, not large enough to neglect finite-chain-size effects, i.e., important properties such as the glass transition temperature $T_{\mathrm{g}}$ or the affine shear modulus $\mu_{\mathrm{A}}$ have not yet reached their $N$-independent asymptotic values $[23,39]$. The total number of monomers $n=N M=12288$ is sufficient to make continuum mechanics applicable. See Sec. VI below for a brief comment on our 


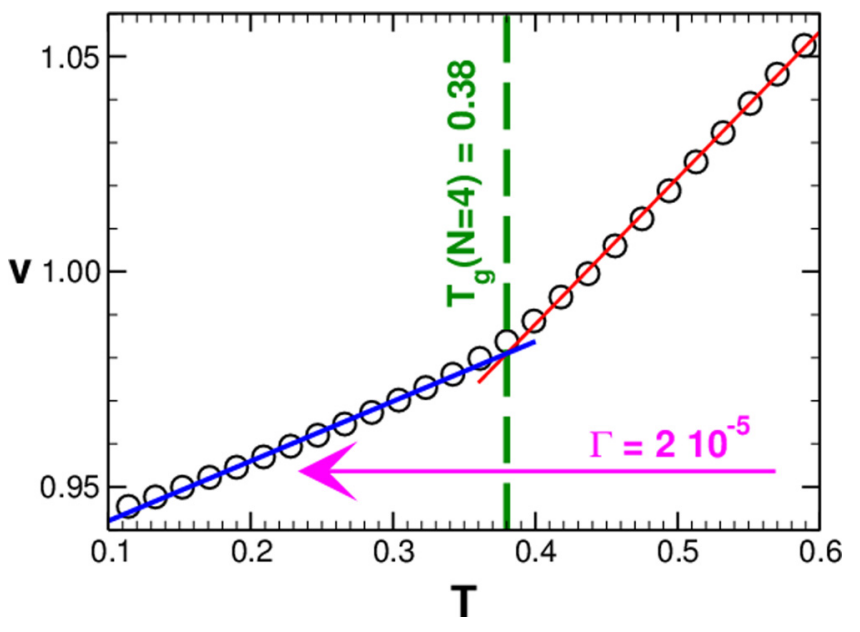

FIG. 2. Average specific volume $v$ per monomer as a function of $T$ for $\Gamma=2 \times 10^{-5}$. The thin solid lines indicate linear fits to the glass and the liquid branches. Using this dilatometric criterion, one defines a glass transition temperature $T_{\mathrm{g}} \approx 0.38$ from the intersection of both linear asymptotes.

ongoing work on system-size effects. The large number $m$ of independent systems allows the precise characterization of ensemble averages, standard deviations, and error bars. For the numerical integration of the equation of motion we use a velocity-Verlet scheme with time steps of length $\delta t_{\mathrm{MD}}=0.005$. The temperature $T$ is imposed by means of the Nosé-Hoover algorithm and the average normal pressure $P$ by the NoséHoover-Andersen barostat (both provided by LAMMPS [21]). All simulations are carried out at $P=0$. Standard cubic simulation boxes with periodic boundary conditions are used throughout this work, i.e., the shape of the box is imposed and does not fluctuate as was the case in recent related studies $[11,29-32]$.

\section{Quench of configuration ensemble}

We start the quench with $m=100$ independent equilibrated configurations at $T=0.6$ and $P=0$. We continuously cool down the configurations with a constant cooling rate $\Gamma=$ $2 \times 10^{-5}$ [39] while keeping constant the average normal pressure $P=0$ letting thus the instantaneous volume $\hat{V}$ of each configuration fluctuate. As may be seen from Fig. 2, the average specific volume $v=\langle\hat{V}\rangle / n \approx 1$ decreases slightly with decreasing temperature $T$. Using the intersection of the linear extrapolations of the glass and the liquid branches of $v$ (or of its logarithm) [2,22,23,39], this provides a simple and experimentally meaningful operational definition of the glass transition temperature $T_{\mathrm{g}}$. We obtain

$$
T_{\mathrm{g}} \approx 0.38 \text { for } P=0, \quad N=4, \text { and } \Gamma=2 \times 10^{-5} .
$$

[As seen from Fig. 5 in Sec. III C, a similar value is obtained from the affine shear modulus $\mu_{\mathrm{A}}(T)$.] After having reached a specific working temperature $T$, the configuration is first tempered over $\Delta t_{\text {temp }}=10^{5}$ at constant pressure [40]. We switch then to the standard canonical ensemble, i.e., the volume $\bar{V}$ of each configuration is fixed, and temper the systems again over $\Delta t_{\text {temp }}$.
TABLE I. Averages obtained for a given shear plane from the time series of instantaneous shear stresses $\hat{\tau}$ and affine shear moduli $\hat{\mu}_{\mathrm{A}}$. The five static properties refer to arithmetic averages obtained over a given time window $\left(t_{1}, t_{2}=t_{1}+\Delta t\right)$ using sampling times $\Delta t \leqslant$ $\Delta t_{\text {max }}$, the two dynamic properties $\bar{c}(t)$ and $\bar{h}(t)$ to the corresponding gliding averages computed over all possible pairs of time $t^{\prime}$ and $t+t^{\prime}$ with $t_{1} \leqslant t^{\prime} \leqslant t+t^{\prime} \leqslant t_{2}$.

\begin{tabular}{lll}
\hline \hline Notation & \multicolumn{1}{c}{ Description } & Definition \\
\hline $\bar{\mu}_{\mathrm{A}}$ & Time-averaged affine shear modulus & Eq. (5) \\
$\bar{\mu}_{0}$ & Time-averaged squared shear stress & Eq. (6) \\
$\bar{\mu}_{1}$ & Squared time-averaged shear stress & Eq. (7) \\
$\bar{\mu}_{\mathrm{F}}$ & Time-averaged shear-stress fluctuation & Eq. (8) \\
$\bar{\mu}_{\mathrm{sf}}$ & Time-averaged shear modulus & Eq. (9) \\
$\bar{c}(t)$ & Gliding-averaged shear-stress ACF & Eq. (10) \\
$\bar{h}(t)$ & Gliding-averaged shear-stress MSD & Eq. (11) \\
\hline \hline
\end{tabular}

\section{Time averages}

The subsequent production runs are performed over $\Delta t_{\max }=10^{5}$ with entries made every $10 \delta t_{\mathrm{MD}}$. Of importance for this study are the instantaneous shear stress $\hat{\tau}$ and the instantaneous "affine shear modulus" $\hat{\mu}_{\mathrm{A}}$ obtained using Eqs. (A6)-(A9) given in Appendix A. As reminded there, $\hat{\tau}$ is the first functional derivative of the Hamiltonian with respect to an imposed infinitesimal canonical and affine shear transformation and $\hat{\mu}_{\mathrm{A}}$ the corresponding second functional derivative. (The Born-Lamé coefficient $\hat{\mu}_{\mathrm{A}}$ is elsewhere also called "affine shear elasticity" or "high-frequency shear modulus" $[11,17,29,32]$.) As summarized in Table I, the stored time series are used to compute for a given configuration and shear plane various time averages (marked by horizontal bars) of instantaneous properties computed over a broad range of sampling times $\Delta t \leqslant \Delta t_{\max }$. Specifically, we shall investigate in Sec. III the following arithmetic averages:

$$
\begin{gathered}
\bar{\mu}_{\mathrm{A}} \equiv \overline{\hat{\mu}_{\mathrm{A}}}, \\
\bar{\mu}_{0} \equiv \beta V \overline{\hat{\tau}^{2}}, \\
\bar{\mu}_{1} \equiv \beta V \overline{\hat{\tau}}^{2}, \\
\bar{\mu}_{\mathrm{F}} \equiv \bar{\mu}_{0}-\bar{\mu}_{1} \geqslant 0, \\
\bar{\mu}_{\mathrm{sf}} \equiv \bar{\mu}_{\mathrm{A}}-\bar{\mu}_{\mathrm{F}} \equiv\left(\bar{\mu}_{\mathrm{A}}-\bar{\mu}_{0}\right)+\bar{\mu}_{1}
\end{gathered}
$$

computed for a given time interval $\left(t_{1}, t_{2}=t_{1}+\Delta t\right)$ with $\beta=$ $1 / T$ being the inverse temperature and $V=\langle\bar{V}\rangle$ the ensembleaveraged volume [41]. $\bar{\mu}_{0}$ measures the $\Delta t$-averaged squared stress, $\bar{\mu}_{1}$ the squared $\Delta t$-averaged stress, and $\bar{\mu}_{\mathrm{F}}$ the rescaled stress fluctuation. The last relation (9) corresponds to the stressfluctuation formula for the shear modulus for one shear plane of a given configuration [1,2,11,12,16,17,22,24-32].

We also consider dynamical properties related to the stress fluctuations of each shear plane such as the shear-stress autocorrelation function (ACF) $\bar{c}(t)$ and the shear-stress meansquare displacement (MSD) $\bar{h}(t)$ being defined, respectively, by

$$
\begin{gathered}
\bar{c}(t) \equiv \beta V \overline{\bar{\tau}\left(t+t^{\prime}\right) \hat{\tau}\left(t^{\prime}\right)} \\
\bar{h}(t) \equiv \frac{\beta V}{2} \overline{\left[\hat{\tau}\left(t+t^{\prime}\right)-\hat{\tau}\left(t^{\prime}\right)\right]^{2}}=\bar{\mu}_{0}-\bar{c}(t) .
\end{gathered}
$$


TABLE II. Some properties as a function of temperature: volume $v$ per monomer, affine shear modulus $\mu_{\mathrm{A}}$ and its standard deviation $\delta \mu_{\mathrm{A}}, \mu_{0}$ and $\delta \mu_{0}, \mu_{1}$ and $\delta \mu_{1}$, shear-stress fluctuation $\mu_{\mathrm{F}}=\mu_{0}-\mu_{1}$ and its standard deviation $\delta \mu_{\mathrm{F}}$, shear-stress modulus $\mu_{\mathrm{sf}}=\mu_{\mathrm{A}}-\mu_{\mathrm{F}}$ and its standard deviation $\delta \mu_{\mathrm{sf}}$, shear-stress relaxation modulus $G(t)$ and its standard deviation $\delta G(t)$ taken at $t=10000$, shear viscosity $\eta_{\infty}$ obtained in the liquid regime using Eq. (2) and terminal relaxation time $\tau_{\infty}$ from the $\mu_{1}(\Delta t)$ scaling (Fig. 17). The data from the 3rd to the 10th columns have been obtained using the largest sampling time $\Delta t=\Delta t_{\max }=10^{5}$. In agreement with Lutsko [26], $\mu_{\mathrm{F}}$ does not vanish for small temperatures and $\mu_{\mathrm{A}}$ is thus only an upper bound to $\mu_{\mathrm{sf}}$ for all temperatures. Importantly, $\mu_{0}$ deviates from $\mu_{\mathrm{A}}$ and $\mu_{1}$ from $\mu_{\mathrm{sf}}$ below $T \approx 0.3$. While $\mu_{\mathrm{A}}, \mu_{0}$, and $G(t)$ do not depend on the sampling time $\Delta t$, this is different for $\mu_{1}, \mu_{\mathrm{F}}, \mu_{\mathrm{sf}}$ and the standard deviations $\delta \mu_{\mathrm{A}}, \delta \mu_{0}, \delta \mu_{1}$, $\delta \mu_{\mathrm{F}}, \delta \mu_{\mathrm{sf}}$, and $\delta G(t)$. See Secs. IIIC and IIID for more details.

\begin{tabular}{|c|c|c|c|c|c|c|c|c|c|c|c|c|c|c|c|}
\hline$T$ & $v$ & $\mu_{\mathrm{A}}$ & $\delta \mu_{\mathrm{A}}$ & $\mu_{0}$ & $\delta \mu_{0}$ & $\mu_{1}$ & $\delta \mu_{1}$ & $\mu_{\mathrm{F}}$ & $\delta \mu_{\mathrm{F}}$ & $\mu_{\mathrm{sf}}$ & $\delta \mu_{\mathrm{sf}}$ & $G(t)$ & $\delta G(t)$ & $\eta_{\infty}$ & $\tau_{\infty}$ \\
\hline 0.30 & 0.9696 & 81.0 & 0.63 & 84.6 & 17.7 & 14.5 & 20.7 & 68.4 & 1.49 & 11.4 & 1.4 & 13.6 & 5.1 & & \\
\hline 0.35 & 0.9777 & 80.2 & 0.62 & 81.6 & 12.4 & 8.5 & 11.9 & 69.4 & 3.47 & 6.8 & 3.5 & 3.9 & 12.6 & & \\
\hline 0.36 & 0.9797 & 80.1 & 0.62 & 80.9 & 9.2 & 6.3 & 9.0 & 69.9 & 3.41 & 6.3 & 3.4 & 5.3 & 11.4 & & \\
\hline 0.37 & 0.9817 & 80.1 & 0.55 & 79.9 & 5.8 & 3.2 & 4.6 & 72.0 & 3.24 & 3.5 & 3.3 & & 12.3 & 275000 & 178000 \\
\hline 0.38 & 0.9838 & 79.7 & 0.55 & 79.7 & 2.6 & 1.0 & 1.5 & 74.8 & 2.19 & 1.1 & 2.2 & & 7.9 & 50000 & 34000 \\
\hline 0.39 & 0.9860 & 79.3 & 0.46 & 79.4 & 1.3 & 0.26 & 0.4 & 77.5 & 1.22 & 0.8 & 0.2 & $\approx 0$ & 7.3 & 13000 & 8300 \\
\hline 0.40 & 0.9888 & 78.6 & 0.31 & 78.9 & 0.70 & 0.09 & 0.1 & 78.0 & 0.68 & $\approx 0$ & 0.7 & $\approx 0$ & 5.5 & 4500 & 4000 \\
\hline 0.41 & 0.9915 & 78.5 & 0.21 & 78.5 & 0.46 & 0.04 & 0.1 & 78.1 & 0.46 & $\approx 0$ & 0.5 & $\approx 0$ & 3.0 & 1800 & 2491 \\
\hline 0.42 & 0.9949 & 78.1 & 0.14 & 78.1 & 0.29 & 0.04 & 0.05 & 77.7 & 0.29 & $\approx 0$ & 0.3 & $\approx 0$ & 2.3 & 890 & 1641 \\
\hline 0.43 & 0.9973 & 77.7 & 0.12 & 77.7 & 0.24 & 0.01 & 0.02 & 77.6 & 0.23 & $\approx 0$ & 0.27 & $\approx 0$ & 1.7 & 460 & 935 \\
\hline 0.44 & 1.0002 & 77.3 & 0.10 & 77.3 & 0.19 & 0.006 & 0.008 & 77.2 & 0.19 & $\approx 0$ & 0.22 & $\approx 0$ & 1.3 & 320 & 712 \\
\hline 0.45 & 1.0040 & 76.7 & 0.08 & 76.9 & 0.17 & 0.004 & 0.005 & 76.9 & 0.17 & $\approx 0$ & 0.19 & $\approx 0$ & 1.2 & 220 & 525 \\
\hline 0.50 & 1.0207 & 74.8 & 0.04 & 74.9 & 0.11 & 0.001 & 0.002 & 74.9 & 0.11 & $\approx 0$ & 0.12 & $\approx 0$ & 0.8 & 59 & 66 \\
\hline 0.55 & 1.0387 & 73.1 & 0.03 & 73.1 & 0.10 & 0.001 & 0.001 & 73.0 & 0.10 & $\approx 0$ & 0.10 & $\approx 0$ & 0.7 & 30 & 26 \\
\hline
\end{tabular}

The bars indicate here that we perform for the time series of each shear plane standard gliding averages [20] over all possible pairs of entries $\hat{\tau}\left(t^{\prime}\right)$ and $\hat{\tau}\left(t^{\prime}+t\right)$ with $0<t_{1} \leqslant t^{\prime} \leqslant$ $t+t^{\prime} \leqslant t_{2}=t_{1}+\Delta t \leqslant \Delta t_{\max }$. This implies that the number of pairs contributing to the gliding average decreases linearly with $t$ and the statistics must thus deteriorate for $t \rightarrow \Delta t$. As reminded in Appendix $\mathrm{C}$, the time-averaged shear-stress relaxation modulus of a given configuration and shear plane is then given in general by $[17,24,29,30,32]$

$$
\bar{G}(t) \equiv \bar{\mu}_{\mathrm{A}}-\bar{h}(t)=\left(\bar{\mu}_{\mathrm{A}}-\bar{\mu}_{0}\right)+\bar{c}(t)
$$

Note that Eq. (12) reduces to the commonly assumed $\bar{G}(t)=$ $\bar{c}(t)[20,42]$ if and only if $\bar{\mu}_{\mathrm{A}}=\bar{\mu}_{0}$ holds.

\section{E. Ensemble averages}

By averaging over the $m$ configurations, the three shear planes and several (at most up to 100) time windows $\left(t_{1}, t_{2}=\right.$ $t_{1}+\Delta t$ ), we obtain then the ensemble averages

$$
\begin{gathered}
\mu_{\mathrm{A}} \equiv\left\langle\bar{\mu}_{\mathrm{A}}\right\rangle \\
\mu_{0} \equiv\left\langle\bar{\mu}_{0}\right\rangle \\
\mu_{1} \equiv\left\langle\bar{\mu}_{1}\right\rangle \\
\mu_{\mathrm{F}} \equiv\left\langle\bar{\mu}_{\mathrm{F}}\right\rangle \\
\mu_{\mathrm{sf}} \equiv\left\langle\bar{\mu}_{\mathrm{sf}}\right\rangle=\mu_{\mathrm{A}}-\mu_{\mathrm{F}}=\left(\mu_{\mathrm{A}}-\mu_{0}\right)+\mu_{1} \\
c(t) \equiv\langle\bar{c}(t)\rangle \\
h(t) \equiv\langle\bar{h}(t)\rangle=\mu_{0}-c(t) \\
G(t) \equiv\langle\bar{G}(t)\rangle=\mu_{\mathrm{A}}-h(t)=\left(\mu_{\mathrm{A}}-\mu_{0}\right)+c(t)
\end{gathered}
$$

denoted by $\langle\ldots\rangle$. Some values are indicated in Table II. As seen from the table and as further discussed in Sec. III C, $\mu_{\mathrm{A}}=$ $\mu_{0}$ for temperatures above $T \approx 0.3$. As one would expect for liquids, this implies that Eqs. (17) and (20) reduce to

$$
\mu_{\mathrm{sf}}=\mu_{1} \text { and } G(t)=c(t) \text { for } T \geqslant 0.3 \text {. }
$$

Table II also contains standard deviations of various observables such as the standard deviation $\delta \mu_{\text {sf }}$ of the shear modulus $\mu_{\text {sf }}$ given by

$$
\delta \mu_{\mathrm{sf}} \equiv \sqrt{\left\langle\bar{\mu}_{\mathrm{sf}}^{2}\right\rangle-\left\langle\bar{\mu}_{\mathrm{sf}}\right\rangle^{2}} .
$$

The error bars are obtained from the indicated standard deviations by dividing by $\sqrt{3 m-1}$ if one assumes the shear planes to be statistically independent (which is a delicate issue) or by $\approx \sqrt{m}$ if one wishes to take a more conservative estimate.

\section{F. Truncation corrections}

Albeit the truncated and shifted LJ potential is continuous, it is not continuous with respect to its first derivative. As can be seen from Eq. (A9), one contribution to $\mu_{\mathrm{A}}$ depends on the second derivative of the potential. Following Ref. [43], impulsive truncation corrections are thus required for the determination of the Born-Lamé coefficient $\mu_{\mathrm{A}}$. These truncation corrections correspond to a shift of about -0.3 for all temperatures. This is taken into account in Table II as elsewhere. In practice, this correction is only relevant for some specific properties at high temperatures. As shown in Appendix B, the shear modulus does otherwise not rigorously vanish as $\mu_{\mathrm{sf}}(\Delta t) \approx \mu_{1}(\Delta t) \sim$ $1 / \Delta t$ to leading order as expected on general grounds related to the well-known finite-sampling-time corrections of timepreaveraged fluctuations [11,17,29-32,44]. 


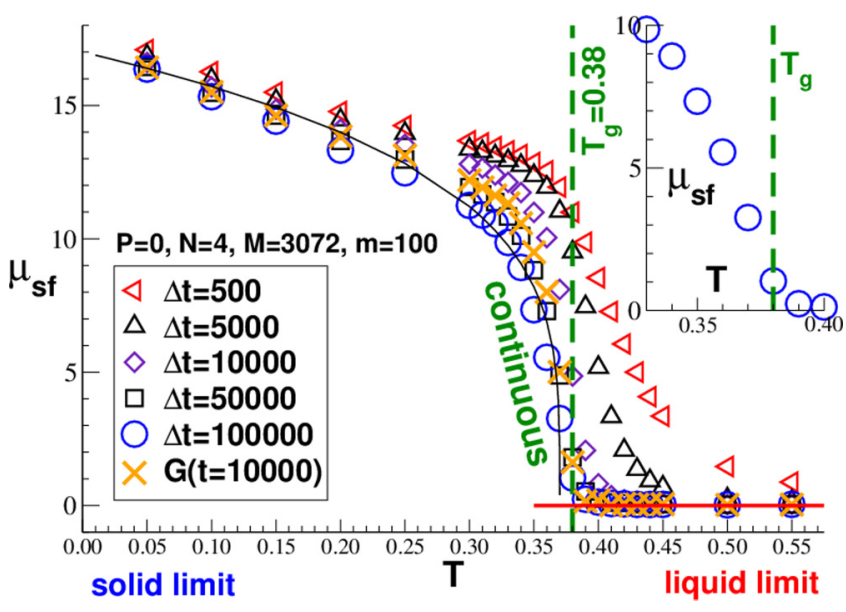

FIG. 3. Shear modulus $\mu_{\mathrm{sf}}(T)=\left\langle\bar{\mu}_{\mathrm{sf}}(T)\right\rangle$ for different sampling times $\Delta t$ using a linear representation. The transition becomes more and more steplike with increasing $\Delta t$ but remains continuous for all $\Delta t$ sampled. Also included is the shear-stress relaxation modulus $G(t)$ taken at a time $t=10^{4}$ (crosses). The vertical dashed line indicates the ( $\Delta t$-independent) glass transition temperature [Eq. (4)], operationally defined using a dilatometric criterion during the continuous temperature quench. The thin solid line corresponds to a cusp singularity with an effective exponent $\alpha \approx 0.2$. Inset: zoom for $T$ around $T_{\mathrm{g}}$ for $\Delta t=100000$ emphasizing that the transition characterized by $\mu_{\mathrm{sf}}(T)$ remains continuous.

\section{EXPECTATION VALUES}

\section{A. Shear modulus $\mu_{\mathrm{sf}}$}

Using a linear representation, we present in Fig. 3 the shear modulus $\mu_{\mathrm{sf}}(T)$ determined by means of the stress-fluctuation relation (17). Data for several sampling times $\Delta t$ are given. There are two points to be emphasized here. First, $\mu_{\mathrm{sf}}(T ; \Delta t)$ depends strongly on $\Delta t$. It is not clear from Fig. 3 whether this dependence may drop out ultimately in the large- $\Delta t$ limit. Second, while the transition becomes systematically more steplike with increasing $\Delta t$, it clearly remains continuous for all $\Delta t$ available. (This is emphasized in the inset of Fig. 3.) The predicted discontinuous jump [4-8] must therefore be strongly blurred by relaxation effects. For large $\Delta t$, our data differ also qualitatively from the parabolic cusp singularity predicted in Ref. [10] and observed by some of the authors in Monte Carlo simulations of two-dimensional polydisperse LJ beads [11]. In fact, as indicated by the thin solid line, a much better phenomenological fit is obtained for $\Delta t=10^{5}$ using

$$
\mu_{\mathrm{sf}}(T) \approx 17\left(1-1.03 T / T_{\mathrm{g}}\right)^{\alpha} \text { with } \alpha \approx 0.2
$$

This effective power-law exponent corresponds to a much stronger increase below the glass transition as predicted by the so-called "disorder-assisted melting" approach $(\alpha=0.5)$ put forward in Ref. [10]. Please note that the indicated fit is only shown to describe the data and no physical meaning should be attributed to the given constants and the exponent $\alpha$. We shall address the observed $\Delta t$ dependence of $\mu_{\mathrm{sf}}$ more systematically in Sec. IIID.

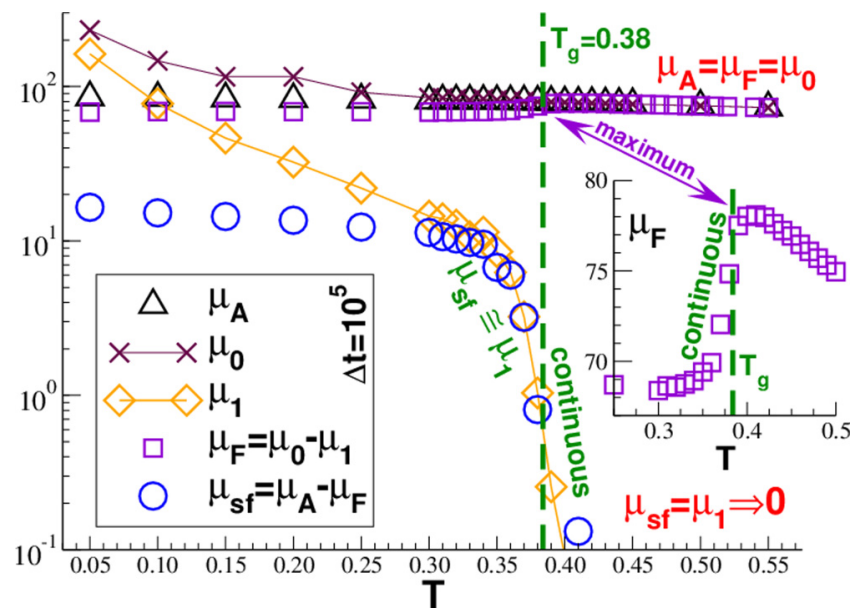

FIG. 4. $\mu_{\mathrm{A}}, \mu_{0}, \mu_{1}, \mu_{\mathrm{F}}$, and $\mu_{\mathrm{sf}}$ vs $T$ using a half-logarithmic representation. Only data for $\Delta t=\Delta t_{\max }=10^{5}$ are given. For large temperatures $\mu_{\mathrm{sf}} \approx \mu_{1}$ and $\mu_{\mathrm{F}} \approx \mu_{0} \approx \mu_{\mathrm{A}}$. With decreasing temperature $\mu_{\mathrm{sf}}$ increases rapidly around $T_{\mathrm{g}}$, but remains continuous. $\mu_{0}$ and $\mu_{1}$ increase rapidly below $T \approx 0.3$ and $\mu_{1}-\mu_{\text {sf }}$ and $\mu_{0}-\mu_{\mathrm{A}}$ become thus finite. Inset: $\mu_{\mathrm{F}}(T)$ using linear coordinates emphasizing the maximum near $T_{\mathrm{g}}$.

\section{B. Related expectation values}

The main panel of Fig. 4 presents $\mu_{\mathrm{sf}}(T)$ and its various contributions for $\Delta t=\Delta t_{\max }=10^{5}$ using half-logarithmic coordinates. As emphasized above, albeit $\mu_{\text {sf }}$ increases rapidly below $T_{\mathrm{g}}$, the data remain continuous in line with findings reported for colloidal glass formers $[1,2,11]$ using also the stress-fluctuation formula. As one expects, $\mu_{\mathrm{sf}} \approx \mu_{1} \rightarrow 0$ in the liquid limit above $T_{\mathrm{g}}$. Using the stress-fluctuation formula $\mu_{\mathrm{sf}}=\mu_{\mathrm{A}}-\mu_{0}+\mu_{1}$, this implies $\mu_{\mathrm{F}}=\mu_{0}=\mu_{\mathrm{A}}[11,24]$. At variance to this, $\mu_{\mathrm{F}}<\mu_{\mathrm{A}}$ below $T_{\mathrm{g}}$, i.e., the stress fluctuations do not have sufficient time to fully explore the phase space. In agreement with Lutsko [26] and more recent studies $[2,11,27,28], \mu_{\mathrm{F}}$ does not vanish for $T \rightarrow 0$, i.e., $\mu_{\mathrm{A}}$ is only an upper bound of $\mu_{\mathrm{sf}}=\mu_{\mathrm{A}}-\mu_{\mathrm{F}}$ for all $T$. Between both $T$ limits, $\mu_{\mathrm{F}}(T)$ has a clear maximum near $T_{\mathrm{g}}$. (This can be better seen using the linear representation given in the inset.) Interestingly, while the difference $\mu_{\mathrm{F}}=\mu_{0}-\mu_{1}$ is more or less constant below $T_{\mathrm{g}}$, its two contributions $\mu_{0}$ and $\mu_{1}$ increase rapidly with decreasing $T$. The reason for this is that strong quenched shear stresses appear which do matter for $\mu_{0}$ and $\mu_{1}$, but nearly cancel out for their difference $\mu_{\mathrm{F}}$. One thus expects much stronger fluctuations between different configurations (and shear planes) for $\mu_{0}$ and $\mu_{1}$ than for $\mu_{\mathrm{F}}$. We shall verify this in Sec. IV C. Interestingly, while $\mu_{0}$ and $\mu_{\mathrm{A}}$ are identical at high temperatures, they become very different below the glass transition. We address this finding in the subsequent subsection.

\section{C. $\Delta t$-independent static properties}

Before we return in Sec. IIID to the sampling time dependence shown in Fig. 3, we need to emphasize that the expectation values of some properties are in fact $\Delta t$ independent. As expected from crystalline and amorphous solids $[2,11]$ and permanent [11,32] and transient [17] elastic networks, this is 


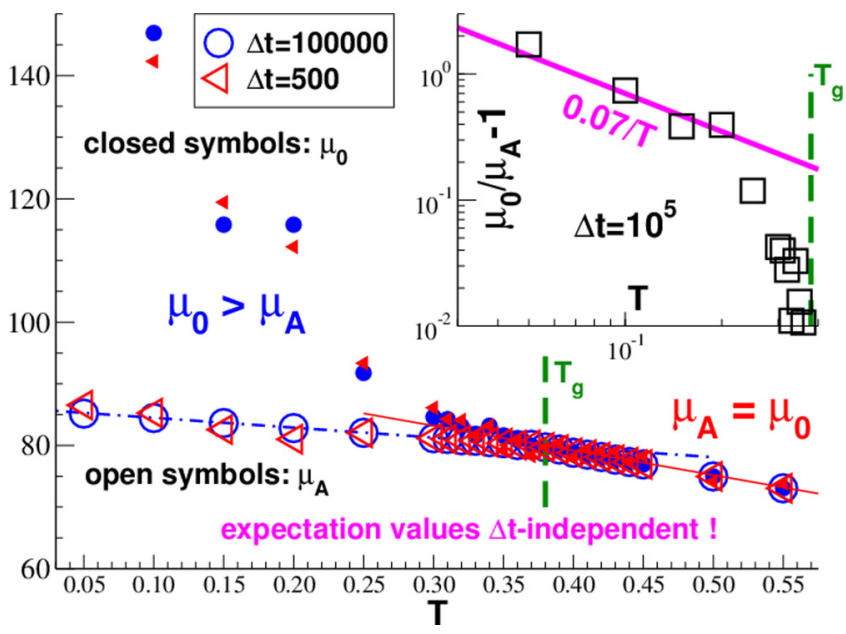

FIG. 5. Temperature dependence of $\mu_{\mathrm{A}}$ and $\mu_{0}$. Main panel: $\mu_{\mathrm{A}}$ (open symbols) and $\mu_{0}$ (closed symbols) for two sampling times illustrating the $\Delta t$ independence expected from the commutation of time and ensemble averages [Eq. (24)]. While $\mu_{\mathrm{A}}(T)$ becomes (more or less) constant below $T_{\mathrm{g}}, \mu_{0}(T)$ is seen to increase strongly. The dashed-dotted and the solid lines indicate two linear fits with $\mu_{\mathrm{A}}(T)=\mu_{\mathrm{A}}\left(T_{\mathrm{g}}\right)\left[1-c\left(T / T_{\mathrm{g}}-1\right)\right]$ with $c=0.076$ and 0.19 for, respectively, the low and high temperature regimes and $T_{\mathrm{g}}=0.38$ for both. Inset: double-logarithmic representation of $\mu_{0} / \mu_{\mathrm{A}}-1$ vs $T$. The ratio decreases inversely with temperature for $T \ll T_{\mathrm{g}}$ (solid line).

the case for $\mu_{\mathrm{A}}$ and $\mu_{0}$. This is demonstrated in Fig. 5 for two sampling times. (We determine first $\bar{\mu}_{\mathrm{A}}$ and $\bar{\mu}_{0}$ from the first $\Delta t$ window of a given time series over $\Delta t_{\max }$ and ensemble average then over $3 m$ shear planes and configurations.) The observed $\Delta t$ independence can be traced back to the fact that their time and ensemble averages commute [32], i.e.,

$$
\langle\overline{\hat{a}(t)}\rangle=\overline{\langle\hat{a}(t)\rangle} \sim \Delta t^{0} \text { since }\langle\hat{a}(t)\rangle \sim \Delta t^{0} .
$$

$\mu_{\mathrm{A}}$ and $\mu_{0}$ are in this sense perfectly defined static observables.

This is of importance since both static properties are seen in Figs. 4 and 5 to behave strikingly different in both temperature limits. We remind that $\mu_{\mathrm{A}}$ is determined solely by the pair correlations of the system while $\mu_{0}$ also contains (in principle) three- and four-point correlations [11]. While in the liquid limit these higher correlations can be factorized (which implies $\left.\mu_{\mathrm{A}}=\mu_{0}\right)$, they become relevant below $T_{\mathrm{g}}$. That $\mu_{0}-\mu_{\mathrm{A}}$ becomes finite below $T_{\mathrm{g}}$ is a nontrivial finding (especially in view of our recent work on transient networks [17]) as shown by the following argument.

Let us suppose that we could have sampled a configuration below (the cooling-rate dependent) $T_{\mathrm{g}}$ over a huge sampling time $\Delta t_{\text {huge }}$ larger than the largest relaxation time $\tau_{\infty}(T)$ of the system. Using the full time series, this would imply that the system must behave as a liquid, i.e., $\mu_{\mathrm{sf}}=0$ and $\mu_{1}=0$. Using the stress-fluctuation formula (17), this implies in turn that $\mu_{0}-\mu_{\mathrm{A}}=0$ if both moments are computed over the full $\Delta t_{\text {huge }}$. However, due to Eq. (24) this must also hold on average for subsets of the complete time series of sampling time $\Delta t \ll$ $\Delta t_{\text {huge }}$. The observation that $\mu_{\mathrm{A}}$ and $\mu_{0}$ systematically deviate below $T_{\mathrm{g}}$ (Fig. 5), thus implies that the $3 m$ time series of length $\Delta t$ obtained from the independently quenched configurations are not equivalent to random subsets of a production run over $\Delta t_{\text {huge }}$.

Basically, $\mu_{0}$ increases much more strongly than $\mu_{\mathrm{A}}$ below $T_{\mathrm{g}}$ due to quenched stresses which do not arise from equilibrium stress fluctuations at the investigated current temperature but at some higher temperature of the quench history. Since $\mu_{0} \sim\left\langle\overline{\hat{\tau}^{2}}\right\rangle / T$ by definition and assuming $\left\langle\overline{\hat{\tau}^{2}}\right\rangle$ to be quenched below $T \approx 0.3$, this suggests that the dimensionless ratio $\mu_{0} / \mu_{\mathrm{A}}-1$ should decay inversely with temperature. Albeit more data points with better statistics are warranted in this limit, this idea is consistent with the inset of Fig. 5.

In summary, due to the quenched shear stresses it is not possible to describe the glassy behavior below $T_{\mathrm{g}}$ by a purely dynamical theory describing the effects of a finite $\Delta t \ll \tau_{\infty}$ under the assumption that the finite time series are randomly drawn from an equilibrium time evolution of a liquid [45]. We note finally that the finding that $\mu_{0} \neq \mu_{\mathrm{A}}$ below $T \approx 0.3$ has important consequences for the numerical determination of the shear-stress relaxation modulus $G(t)$ [24,32]. This point is addressed in Appendix C.

\section{D. $\Delta t$-dependent quasistatic properties}

While time and ensemble averages do commute for $\mu_{\mathrm{A}}$ and $\mu_{0}$, Eq. (24) does not hold for $\mu_{1}, \mu_{\mathrm{F}}$, and $\mu_{\mathrm{sf}}$. We remind that even for permanent elastic networks these observables are known to depend on $\Delta t[11,17,29]$. Since

$$
\mu_{\mathrm{sf}}(\Delta t)=\mu_{\mathrm{A}}-\mu_{\mathrm{F}}(\Delta t)=\left(\mu_{\mathrm{A}}-\mu_{0}\right)+\mu_{1}(\Delta t),
$$

we can focus here on the $\Delta t$ dependence of $\mu_{\mathrm{sf}}(\Delta t)$ as shown in Fig. 6. Covering a broad range of temperatures, we use subsets of length $\Delta t$ of the total trajectories of length $\Delta t_{\max }$ stored. It is seen that $\mu_{\mathrm{sf}}(\Delta t)$ decreases both monotonically and continuously with $\Delta t$. The figure reveals that $\mu_{\mathrm{sf}}(\Delta t ; T)$ decreases also monotonically and continuously with $T$. Note that $\mu_{\mathrm{sf}}(\Delta t)$ increases for $T \rightarrow 0$ while its $\Delta t$ dependence

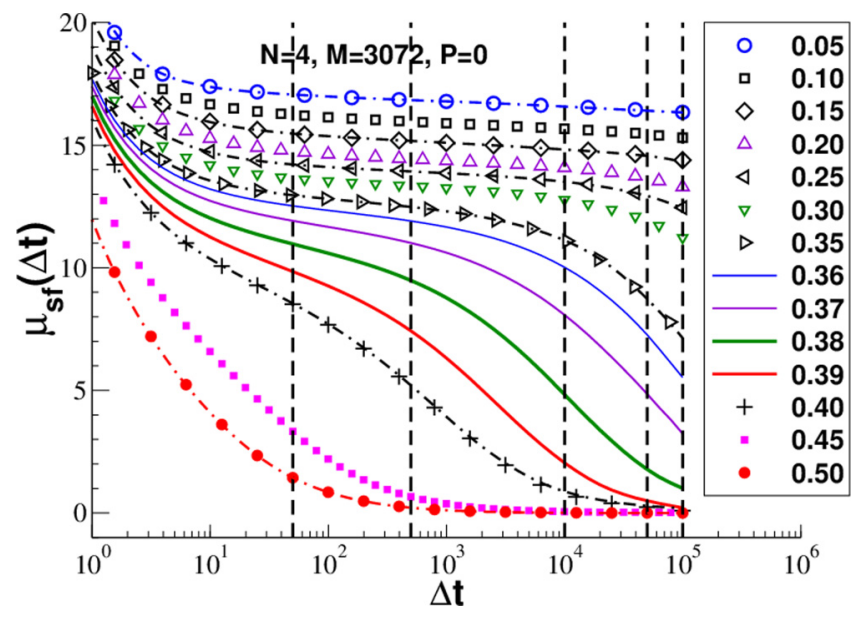

FIG. 6. Shear modulus $\mu_{\mathrm{sf}}$ as a function of sampling time $\Delta t$ for a broad range of $T$ as indicated in the figure. $\mu_{\mathrm{sf}}(\Delta t)$ decreases continuously with $\Delta t$. Note that a smaller temperature increment $\Delta T=0.01$ is used around $T_{\mathrm{g}}$ (solid lines) where $\mu_{\mathrm{sf}}(\Delta t ; T)$ changes much more rapidly with $T$. The vertical lines mark the sampling times used in Fig. 3. The dashed-dotted lines are obtained using Eq. (1) by integrating the shear-stress relaxation modulus $G(t)$. 
becomes weaker. A glance at Fig. 6 shows that one expects the transition of $\mu_{\mathrm{sf}}(T ; \Delta t)$ to get shifted to lower $T$ and to become more steplike with increasing $\Delta t$ in agreement with Fig. 3. It is, however, impossible to reconcile the data with a jump singularity at a finite $\Delta t$ and $T$. As announced in the Introduction, this is the first key result of this work. See Appendix B for the discussion of the technical importance of $\mu_{\mathrm{sf}}(\Delta t) \approx \mu_{1}(\Delta t)$ at high temperatures.

\section{STANDARD DEVIATIONS, DISTRIBUTIONS, AND CORRELATIONS}

\section{A. Standard deviation $\delta \mu_{\mathrm{sf}}$}

To characterize the fluctuations between different configurations we take for various properties the second moment over the ensemble and compute, e.g., the standard deviation $\delta \mu_{\text {sf }}$ of the shear modulus [Eq. (22)]. As seen in Fig. 7, at variance to the monotonic modulus $\mu_{\mathrm{sf}}(T)$ its standard deviation $\delta \mu_{\mathrm{sf}}(T)$ is nonmonotonic with a remarkable peak near $T_{\mathrm{g}}$. (As may be seen from Fig. 8 of Ref. [17], similar behavior has been observed for systems of self-assembled transient networks.) Note that while $\delta \mu_{\text {sf }}(T)$ is essentially $\Delta t$ independent above $T_{\mathrm{g}}$, it increases systematically with $\Delta t$ below the transition. Importantly, the peak of $\delta \mu_{\mathrm{sf}}(T)$ becomes about a third of the drop of the ensemble-averaged shear modulus $\mu_{\mathrm{sf}}(T)$ between $T=0.34$ and 0.38 for $\Delta t=10^{5}$ (cf. Fig. 3). The liquid-solid transition characterized by $\mu_{\mathrm{sf}}(T)$ is thus accompanied by strong fluctuations between different quenched configurations. We note finally that the relative standard deviation $\delta \mu_{\mathrm{sf}}(\Delta t) / \mu_{\mathrm{sf}}(\Delta t)$ is for all temperatures found to increase with $\Delta t$ (not shown). In the solid limit this is due to the increase of $\delta \mu_{\mathrm{sf}}(\Delta t)$, and in the liquid limit due to the $1 / \Delta t$ decay of $\mu_{\mathrm{sf}}(\Delta t)$ discussed in Appendix B and for temperatures around $T_{\mathrm{g}}$ due to a combination of both effects.

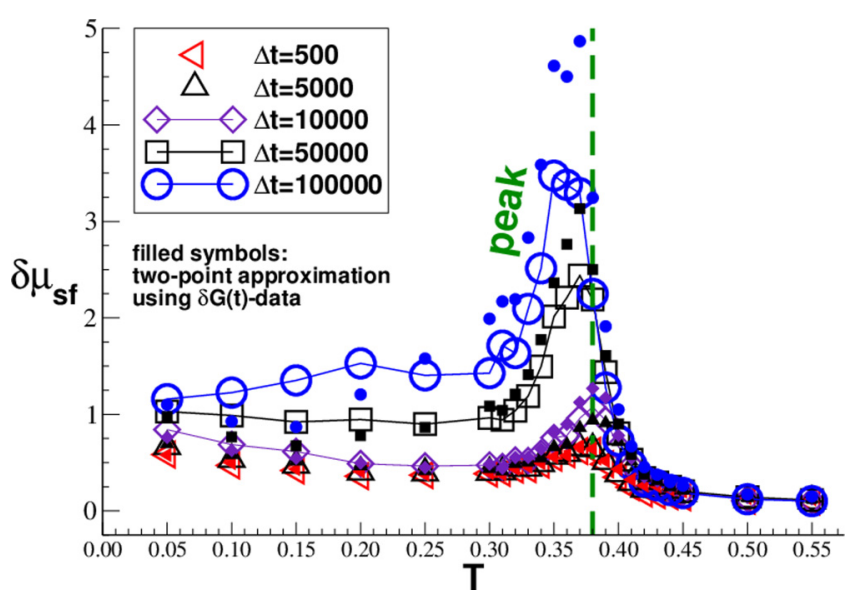

FIG. 7. Standard deviation $\delta \mu_{\mathrm{sf}}(T)$ for different sampling times $\Delta t$ using a linear representation. The observed peak slightly below $T_{\mathrm{g}}$ becomes sharper with increasing $\Delta t$. The small filled symbols indicate the values predicted (Sec. VE) according to the two-point approximation (39) from the standard deviation $\delta G(t)$. While this allows to relate $\mu_{\mathrm{sf}}(\Delta t)$ to $\delta G(t)$ for $T \ll T_{\mathrm{g}}$ and $T \gg T_{\mathrm{g}}$, it fails for large $\Delta t$ around the glass transition.
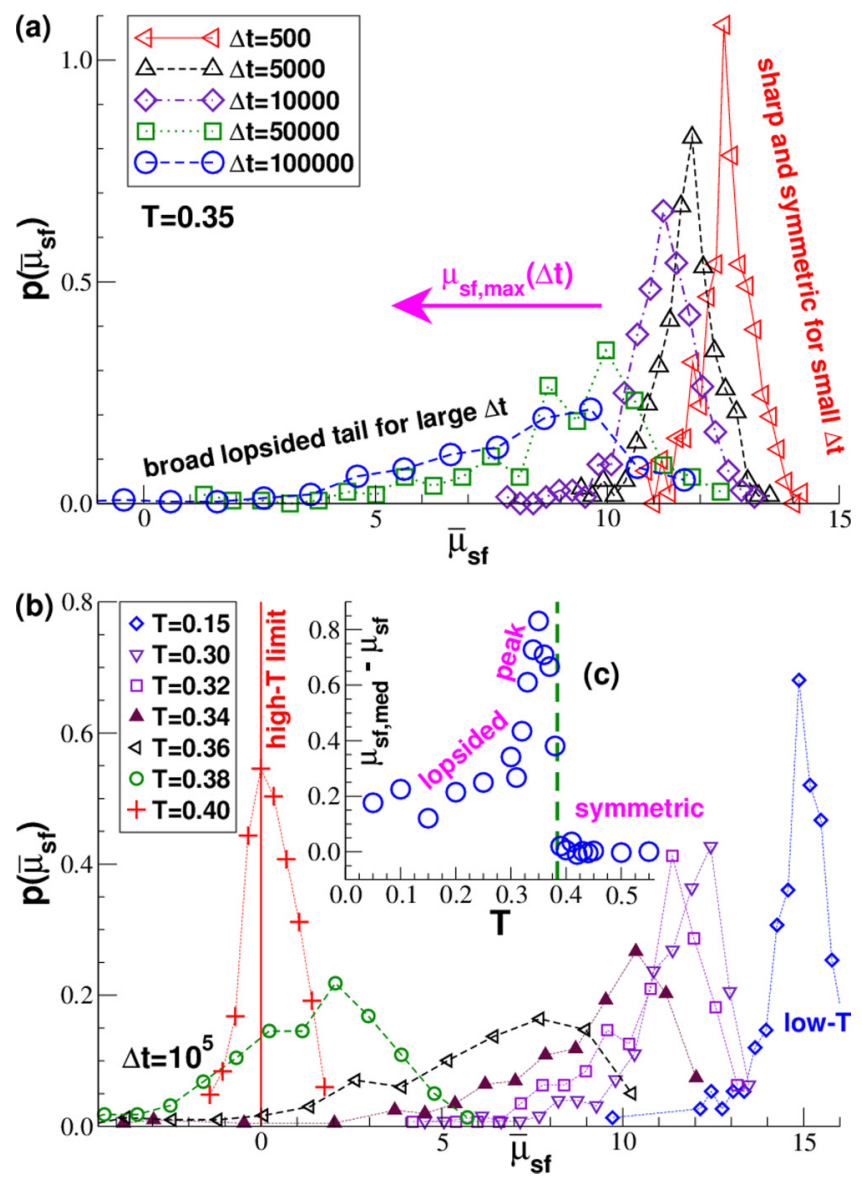

FIG. 8. Distribution $p\left(\bar{\mu}_{\mathrm{sf}}\right)$ of the time-averaged modulus $\bar{\mu}_{\mathrm{sf}}$ : (a) $p\left(\bar{\mu}_{\text {sf }}\right)$ for $T=0.35$ and several $\Delta t$ as indicated. The maximum $\mu_{\mathrm{sf} \text {, max }}$ shifts to the left with increasing $\Delta t$ and the histogram becomes more lopsided. (b) $p\left(\bar{\mu}_{\mathrm{sf}}\right)$ for $\Delta t=10^{5}$ and a broad range of $T$. (c) $\mu_{\mathrm{sf}, \text { med }}-\mu_{\mathrm{sf}}$ vs $T$ for $\Delta t=10^{5}$.

\section{B. Distribution of $\overline{\boldsymbol{\mu}}_{\mathrm{sf}}$}

The striking peak of $\delta \mu_{\mathrm{sf}}$ near $T_{\mathrm{g}}$ seen in Fig. 7 begs for a more detailed characterization of the distribution $p\left(\bar{\mu}_{\mathrm{sf}} ; T, \Delta t\right)$ of the time-averaged shear modulus $\bar{\mu}_{\text {sf }}$. Using the available $3 \times m=300$ independent measurements of $\bar{\mu}_{\text {sf }}$ this is presented in Fig. 8. We emphasize first of all that the histograms are unimodal for all $T$ and $\Delta t$. The $T$ dependence of $\mu_{\mathrm{sf}}$ and $\delta \mu_{\text {sf }}$ below $T_{\mathrm{g}}$ is thus not due to, e.g., the superposition of two configuration populations representing either solid states with finite $\bar{\mu}_{\text {sf }}$ and liquid states with $\bar{\mu}_{\text {sf }} \approx 0$. While the distributions depend only weakly (if at all) on $\Delta t$ in the liquid limit (not shown), the distributions become systematically broader and more lopsided with increasing $\Delta t$ below the glass transition temperature $T_{\mathrm{g}}$ as seen in Fig. 8(a) for $T=0.35$. This explains the increase of $\delta \mu_{\mathrm{sf}}$ with $\Delta t$ seen in Fig. 7. Concurrently, the maximum $\mu_{\mathrm{sf}, \max }$ and the median $\mu_{\mathrm{sf}, \text { med }}$ decrease systematically with increasing $\Delta t$. Both trends are caused by the higher probability of plastic rearrangements if a configuration is probed over a larger time interval. Focusing on our largest sampling time $\Delta t_{\max }$, Fig. 8(b) presents data for a broad range of temperatures. The maximum $\mu_{\mathrm{sf} \text {, max }}$ of the (unimodal) distribution systematically shifts to higher values below $T_{\mathrm{g}}$, in agreement with its first moment $\mu_{\mathrm{sf}}$ (Fig. 3), 
while the distributions become systematically broader and more lopsided, i.e., liquidlike configurations with small $\bar{\mu}_{\text {sf }}$ become relevant. For even smaller temperatures $T \ll T_{\mathrm{g}}$, the distributions get again more focused around $\mu_{\mathrm{sf} \text {,max }}$ and less lopsided in agreement with Fig. 7. That the large standard deviations and the asymmetry of the distributions are related is demonstrated by comparing the first moment $\mu_{\text {sf }}$ of the distribution, its median $\mu_{\mathrm{sf}, \operatorname{med}}$, and its maximum $\mu_{\mathrm{sf}, \max }$. One confirms that

$$
0<\mu_{\mathrm{sf}, \text { med }}-\mu_{\mathrm{sf}}<\mu_{\mathrm{sf}, \max }-\mu_{\mathrm{sf}} \text { for } T<T_{\mathrm{g}}
$$

and for all $\Delta t$. As seen in Fig. 8(c), the difference $\mu_{\text {sf,med }}-\mu_{\text {sf }}$ has a peak similar to $\delta \mu_{\mathrm{sf}}$ slightly below $T_{\mathrm{g}}$ corresponding to very lopsided distributions.

\section{Comparison of related standard deviations}

Using a half-logarithmic representation $\delta \mu_{\mathrm{sf}}$ is replotted in Fig. 9(a) together with the corresponding standard deviations $\delta \mu_{\mathrm{A}}, \delta \mu_{0}, \delta \mu_{1}$, and $\delta \mu_{\mathrm{F}}$. Please note that for all these standard deviations ensemble and time averages do not commute, i.e., these properties depend in principle on the sampling time as we have already seen for $\delta \mu_{\text {sf }}$ in Fig. 7. Another example is given for $\delta \mu_{1}$ in Fig. 9(b) showing that the deviations decrease more rapidly for larger temperatures with increasing $\Delta t$. The logarithmic scale used for the vertical axis masks somewhat the effect better visible in linear coordinates. Returning to Fig. 9(a) we emphasize first of all that $\delta \mu_{\mathrm{A}}$ is negligible and $\delta \mu_{\mathrm{sf}} \approx \delta \mu_{\mathrm{F}}$ for all $T$. In the high- $T$ regime, we find $\delta \mu_{\mathrm{sf}} \approx \delta \mu_{0}$ while $\delta \mu_{1}$ vanishes much more rapidly. Interestingly, in the opposite glass limit $\delta \mu_{\mathrm{sf}} \approx \delta \mu_{\mathrm{F}}$ becomes orders of magnitude smaller than $\delta \mu_{0} \approx \delta \mu_{1}$. The contributions $\bar{\mu}_{0}$ and $\bar{\mu}_{1}$ of the difference $\bar{\mu}_{\mathrm{F}}=\bar{\mu}_{0}-\bar{\mu}_{1}$ thus must be strongly correlated.

\section{Correlations between $\bar{\mu}_{0}$ and $\bar{\mu}_{1}$}

This can be directly verified using the corresponding dimensionless correlation coefficient

$$
r_{01} \equiv \frac{\left\langle\left(\bar{\mu}_{0}-\mu_{0}\right)\left(\bar{\mu}_{1}-\mu_{1}\right)\right\rangle}{\delta \mu_{0} \delta \mu_{1}} .
$$

As can be seen from Fig. 10, $r_{01}(T, \Delta t)$ provides an operationally simple and clear-cut order parameter between the liquid limit $\left(r_{01} \rightarrow 0\right)$ and the solid regime $\left(r_{01} \rightarrow 1\right)$. The latter limit is another manifestation of the frozen shear stresses. Quite generally, one may write the variance $\delta \mu_{\mathrm{F}}^{2}$ of $\mu_{\mathrm{F}}=$ $\mu_{0}-\mu_{1}$ as

$$
\delta \mu_{\mathrm{F}}^{2}=\delta \mu_{0}^{2}+\delta \mu_{1}^{2}-2 r_{01} \delta \mu_{0} \delta \mu_{1} .
$$

Since $r_{01} \approx 1^{-}$for $T \ll T_{\mathrm{g}}$, we have $\delta \mu_{\mathrm{F}} \approx \delta \mu_{0}-\delta \mu_{1} \geqslant 0$. This explains why

(i) $\delta \mu_{\mathrm{F}}$ becomes very small albeit the fluctuations of its contributions $\mu_{0}$ and $\mu_{1}$ are large (Fig. 9) and

(ii) the stress-fluctuation formula (17) for the shear modulus remains a statistically successful approach in the solid limit despite the fact that violent stress fluctuations occur between different configurations of the ensemble.

Please note also that the correlation coefficient $r_{01}(T)$ is again continuous and does depend somewhat on the sampling time $\Delta t$. This $\Delta t$ dependence may be characterized as shown in the inset of Fig. 10 by means of a temperature $T_{1 / 2}$
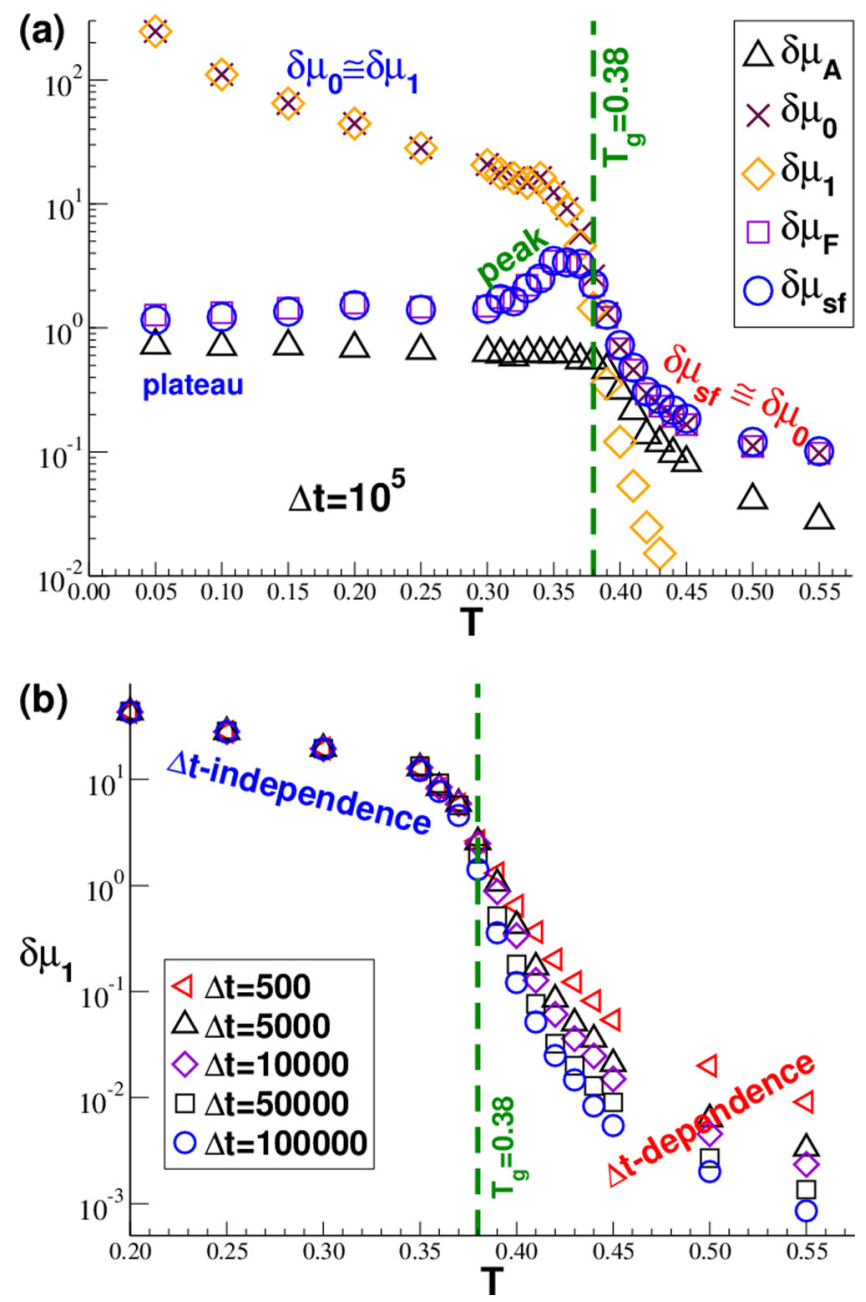

FIG. 9. Standard deviations as a function of $T$ : (a) $\delta \mu_{\mathrm{A}}, \delta \mu_{0}, \delta \mu_{1}$, $\delta \mu_{\mathrm{F}}$, and $\delta \mu_{\mathrm{sf}}$ for $\Delta t=10^{5} . \delta \mu_{\mathrm{A}}$ is found to be small and $\delta \mu_{\mathrm{sf}} \approx \delta \mu_{\mathrm{F}}$ for all $T . \delta \mu_{0}$ and $\delta \mu_{1}$ become rapidly similar below $T_{\mathrm{g}}$ and orders of magnitude larger than $\delta \mu_{\mathrm{F}}$ confirming the presence of strong frozen shear stresses. (b) $\delta \mu_{1}(T)$ for several $\Delta t$ using the same symbols as in Fig. 7 for $\delta \mu_{\mathrm{sf}}(\Delta t)$. While sampling time effects are seen to be irrelevant for $T \ll T_{\mathrm{g}}$, i.e., the frozen stresses cannot relax, they matter for temperatures around and above $T_{\mathrm{g}}$.

defined by $r_{01}\left(T_{1 / 2}, \Delta t\right)=\frac{1}{2}$. It is seen that $T_{1 / 2}(\Delta t)$ decays logarithmically with $\Delta t$, at least for the $\Delta t$ range we are able to probe. Longer production runs are warranted to clarify whether $T_{1 / 2}(\Delta t)$ continues to decrease as we strongly expect.

\section{SHEAR-STRESS RELAXATION MODULUS}

\section{A. Qualitative description of $G(t)$}

The shear-stress relaxation modulus $G(t)$ is an experimentally important observable [46-48]. As shown in Fig. 11, we have computed $G(t)$ by means of the fluctuation-dissipation relation (20) appropriate for canonical ensembles with quenched or sluggish shear stresses [24,32]. This allows us to also sample $G(t)$ below $T_{\mathrm{g}}$ where $\mu_{0}-\mu_{\mathrm{A}}$ becomes finite as shown in Sec. IIIC. (See also Appendix C.) Since $G(t)$ is obtained by means of gliding averages along the time series [Eq. (12)], the statistics deteriorates for $t \rightarrow \Delta t$. [This will be quantified 


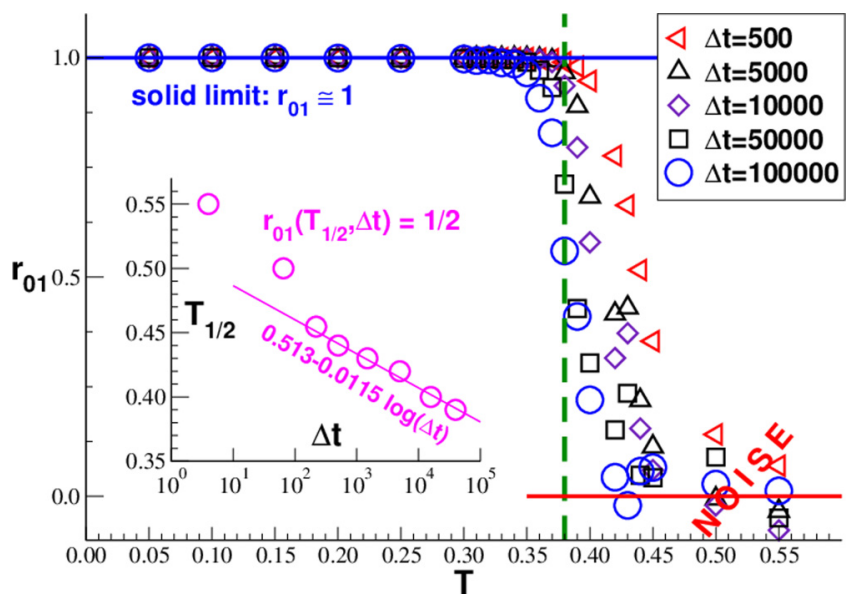

FIG. 10. Correlation coefficient $r_{01}$ as a function of $T$ for different $\Delta t$ showing that $r_{01} \rightarrow 0$ for $T \gg T_{\mathrm{g}}$ and $r_{01} \approx 1$ for $T \ll T_{\mathrm{g}}$. The transition around $T \approx T_{\mathrm{g}}$ depends again on $\Delta t$. Inset: $T_{1 / 2}(\Delta t)$ defined by $r_{01}\left(T_{1 / 2}, \Delta t\right)=\frac{1}{2}$ reveals a logarithmic decay of the correlations with increasing $\Delta t$.

in Sec. VE where we discuss the standard deviation $\delta G(t)$.] We have thus logarithmically averaged the data presented in Fig. 11. This suppresses somewhat the oscillations at small times $t \ll 10$, which are, however, irrelevant for the present study. We emphasize the following qualitative properties:

(a) As it should, $G(t)$ vanishes for large times $t \gg \tau_{\infty}(T)$ in the liquid limit above $T_{\mathrm{g}}$.

(b) $G(t)$ increases monotonically and continuously with decreasing temperature.

(c) This increase is, however, especially strong around $T_{\mathrm{g}}$ where the solid lines indicate, as in Fig. 6, a smaller temperature increment $\Delta T=0.01$.

(d) Albeit we average over $m=100$ configurations and three shear planes, $G(t)$ remains rather noisy for $T \approx T_{\mathrm{g}}$ and $t>10^{4}$. (See Sec. V E for details.)

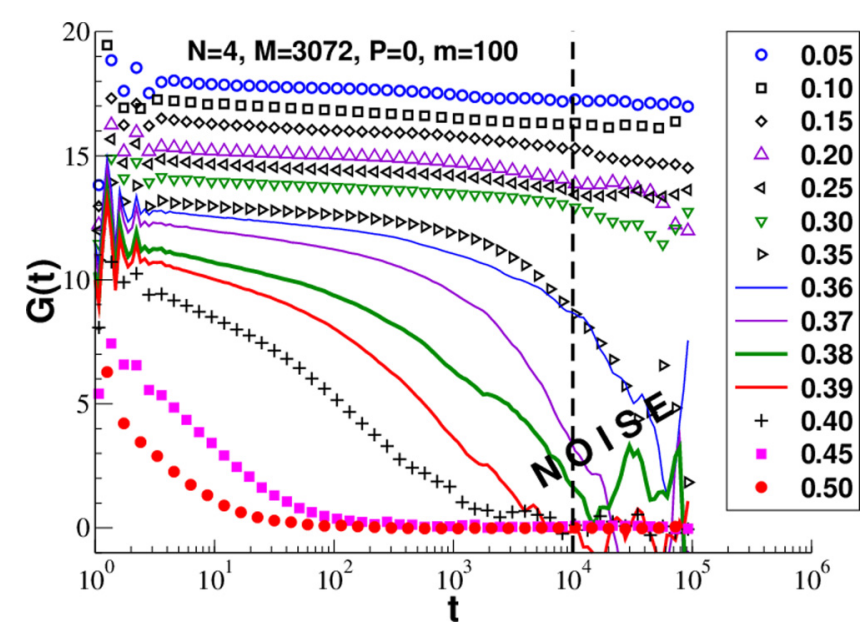

FIG. 11. Stress relaxation modulus $G(t)$ for a broad range of $T$ using half-logarithmic coordinates. $G(t)$ has been obtained by means of Eq. (20) using gliding averages, i.e., the statistics deteriorates for $t \rightarrow \Delta t$ and the data have been logarithmically averaged for clarity. The dashed vertical line marks the time used for $G(t)$ in Fig. 3 and Table II.
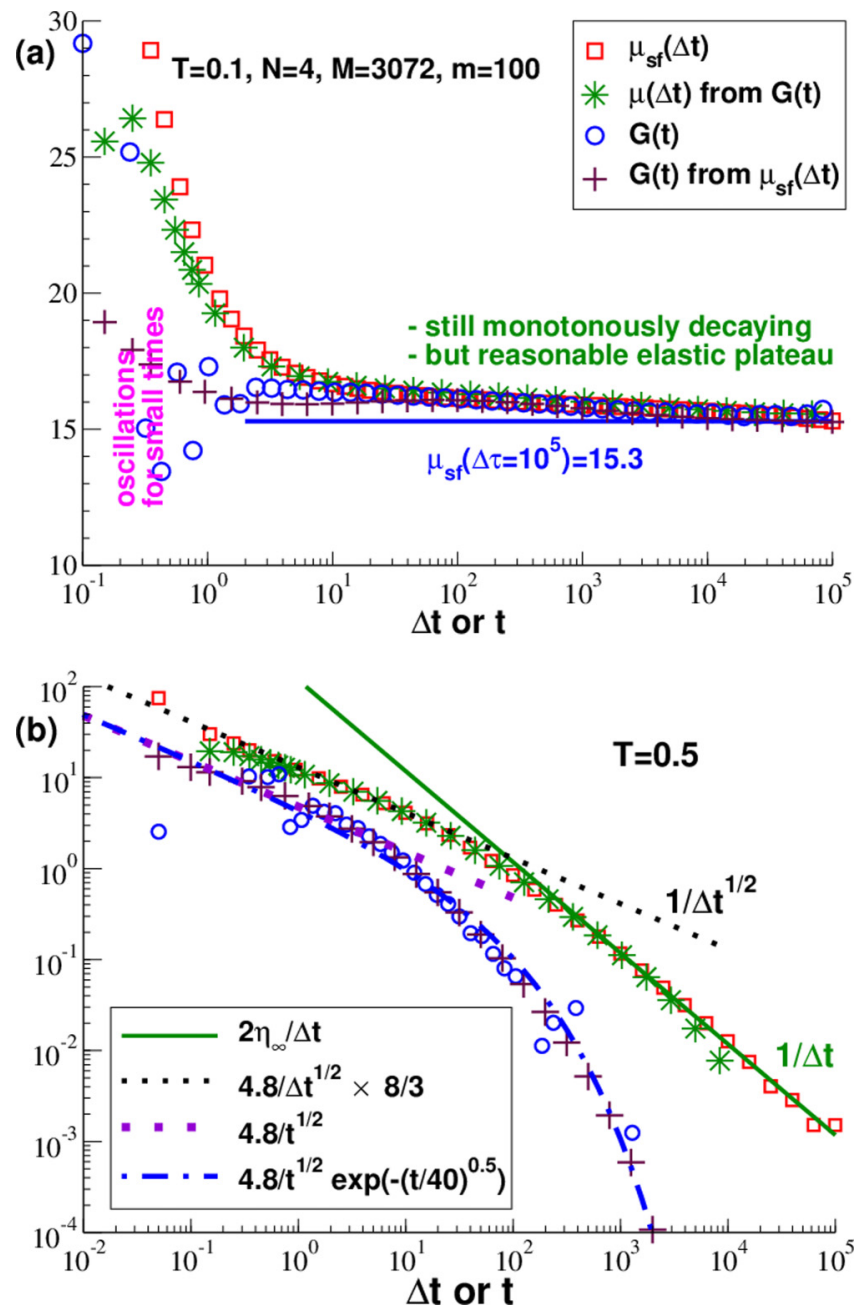

FIG. 12. Comparison of $\mu_{\mathrm{sf}}(\Delta t), \mu(\Delta t)$, and $G(t)$ for $T=0.1$ and 0.5. $\mu_{\mathrm{sf}}(\Delta t)$ is indicated by squares, $\mu(\Delta t)$ by stars, and $G(t)$ by open circles if obtained using Eq. (20) and by pluses if obtained using Eq. (30) from the $\mu_{\mathrm{sf}}(\Delta t)$ data. It is seen that $\mu_{\mathrm{sf}}(\Delta t) \approx \mu(\Delta t)$ and that also both expressions for $G(t)$ are essentially identical. (a) At sufficiently low temperatures $\mu(\Delta t) \approx G(t=\Delta t)$ over a broad plateau. (b) At larger temperatures and longer times $G(t)$ decays faster than its integral $\mu(\Delta t)$. The dotted lines indicate $\mu(\Delta t) \sim 1 / \Delta t^{\alpha}$ and $G(t) \sim 1 / t^{\alpha}$ with $\alpha=\frac{1}{2}$.

(e) $G(t)$ decreases only weakly within the available time window below $T=0.3$.

The data presented in Fig. 11 are thus qualitatively very similar to the shear modulus $\mu_{\mathrm{sf}}(\Delta t)$ given in Fig. 6. We describe now $G(t)$ more quantitatively by focusing on the $\Delta t$-dependent moments $\mu(\Delta t)$ and $\eta(\Delta t)$ defined in the Introduction.

\section{B. Connection between $\mu_{\mathrm{sf}}(\Delta t)$ and $G(t)$}

Using the generic $\Delta t$ dependence of time-averaged fluctuations [44], we relate now the sampling time dependence of $\mu_{\mathrm{sf}}(\Delta t)$, shown in Figs. 6 and 12, to the time dependence of the relaxation modulus $G(t)$. As shown in Appendix D, assuming time-translational invariance $\mu_{\mathrm{sf}}(\Delta t)$ should be equivalent to the weighted average $\mu(\Delta t)$ introduced in Eq. (1) in the Introduction. Using the directly determined $G(t)$ described 
in Sec. VA, one readily computes $\mu(\Delta t)$ as shown by the thin dashed-dotted lines indicated in Fig. 6 and the stars in Fig. 12 for one very low $(T=0.1)$ and one very high $(T=$ $0.5)$ temperature. Since $\mu_{\mathrm{sf}}(\Delta t) \approx \mu(\Delta t)$ is found to high accuracy for all $T$, this confirms the assumed time-translational invariance. The $\Delta t$ dependence of $\mu_{\mathrm{sf}}, \mu_{1}$, and $\mu_{\mathrm{F}}$ is thus simply due to the upper bound $\Delta t$ used to average the relaxation modulus $G(t)$. It is not due to aging or equilibration problems, but to the finite time needed for $G(t)$ to reach its asymptotic limit. This confirms the third key result of this work announced in the Introduction. Since $\mu_{\mathrm{sf}}$ and $\mu$ are identical within numerical accuracy, we often drop the notation $\mu_{\mathrm{sf}}$ below.

\section{Further consequences}

Using integration by parts, it is seen that the definition (1) is equivalent to

$$
\mu(\Delta t)=\frac{2}{\Delta t^{2}} \int_{0}^{\Delta t} d t \int_{0}^{t} d t^{\prime} G\left(t^{\prime}\right) .
$$

This implies that

$$
\begin{aligned}
G(t) & =\left[t^{2} \mu(t) / 2\right]^{\prime \prime} \\
& =\mu(t)+2 t \mu^{\prime}(t)+t^{2} \mu^{\prime \prime}(t) / 2,
\end{aligned}
$$

where a prime denotes a derivative with respect to the argument. This suggests that one may use the smooth $\mu_{\mathrm{sf}}(\Delta t)$ presented in Fig. 6 to compute $G(t)$. This can be done by fitting first to sixth order $y \equiv \ln \left(\mu_{\mathrm{sf}}(\Delta t) \Delta t^{2} / 2\right)$ as a function of $x \equiv \ln (\Delta t)$ and tracing then

$$
G(t)=e^{y(x)-2 x}\left\{y^{\prime \prime}(x)+y^{\prime}(x)\left[y^{\prime}(x)-1\right]\right\} .
$$

As indicated by the pluses in Fig. 12, this yields essentially identical results as the directly computed $G(t)$, however, with much less noise, especially for large $t$. Since $\mu_{\mathrm{A}}=\mu_{0}$ for high temperatures (Fig. 5), we recommend to replace in this limit $\mu_{\mathrm{sf}}(\Delta t)$ by $\mu_{1}(\Delta t)$ to avoid the impulsive corrections discussed in Appendix B.

We emphasize that albeit $\mu(\Delta t)$ and $G(t)$ are similar for all $T$, as may be seen from Fig. 12 and by comparing Figs. 6 and 11 , both quantities are only identical if according to Eq. (31), the second and the third term in Eq. (31) are negligible compared to the first one. As may be seen from Fig. 12(a), this is the case in the solid limit where $G(t)$ may be well approximated by a constant $\mu_{\star}(T)$. Equation (1) thus implies

$$
G(t) \approx \mu(\Delta t) \approx \mu_{\star}(T) \text { for } T \leqslant 0.2 .
$$

See the discussion around Eq. (D2) in Appendix D. This becomes different for higher temperatures where relaxation processes become much more important. As seen for one temperature in Fig. 12(b),

$$
\mu(\Delta t)>G(t \approx \Delta t) \text { for } T>0.2 \text { and } \Delta t \ll \tau_{\infty} .
$$

The inequality is due to the strong weight of small times $t \ll \Delta t$ in Eq. (1). $\mu(\Delta t)$ converges thus more slowly to any intermediate plateau or the asymptotic limit $\left(\Delta t \gg \tau_{\infty}\right)$ as the relaxation modulus $G(t)$. A particular interesting case (not only from the polymer physics point of view) arises if the relaxation modulus does not have an intrinsic time scale over a sufficiently broad time window and decays as a power law $G(t) \sim 1 / t^{\alpha}$ with $1>\alpha>0$. Using Eq. (30) it is seen that $\mu(\Delta t)$ decays with the same exponent $\alpha$ and the relative amplitudes are given by

$$
\frac{\mu(\Delta t)}{G(t=\Delta t)}=\frac{2}{(1-\alpha)(2-\alpha)}=\frac{8}{3} \text { for } \alpha=1 / 2 .
$$

The ratio $\frac{8}{3}$ is consistent with the two dotted power-law slopes indicated in Fig. 12(b). Note that our chains are too short to reveal a full Rouse dynamics [46] and the observed $\alpha=\frac{1}{2}$ is due to the superposition of various different effects (e.g., small- $N$ corrections, crossover from the short-time dynamics to the terminal relaxation). The point we want to make here is merely that whenever scale-free physics characterized by an exponent $\alpha<1$ arises, this implies that $\mu(\Delta t) / G(t \approx \Delta t)$ must be a constant larger than unity.

To summarize, $\mu(\Delta t)=\mu_{\mathrm{sf}}(\Delta t)$ only corresponds to a classical thermodynamic (and thus $\Delta t$ independent) shear (storage) modulus if $G(t)$ becomes essentially constant over a broad time window and if the sampling time $\Delta t$ is sufficiently large to probe this window. In all other cases, $\mu(\Delta t)$ should be seen as a generalized shear modulus or a strong smoothing function over $G(t)$ containing also information from dissipation processes. That this is indeed the case will be shown in Sec. VD.

\section{Shear viscosity $\eta(\Delta t)$}

Standard operational definition. We have shown in Sec. V B that (at least for the glass-forming polymer model investigated) the stress-fluctuation formula (17) for the shear modulus is equivalent to the moment $\mu(\Delta t)$ over $G(t)$ [Eq. (1)]. In order to further describe the relaxation modulus $G(t)$, it is convenient to compute the generalized ( $\Delta t$-dependent) shear viscosity $\eta(\Delta t)$ defined by Eq. (3) in the Introduction. As shown by the open symbols in Fig. 13, we have thus computed $\eta$ both as a function of $\Delta t$ and as a function of $T$. As emphasized by the bold solid line in Fig. 13(a), $\eta(\Delta t ; T) \approx \mu_{\star}(T) \Delta t$ in the solid limit where $G(t) \approx \mu_{\star}(T)$ becomes constant. As one also expects, $\eta(\Delta t ; T)$ levels off, i.e., becomes $\Delta t$ independent for sufficiently high temperatures where $\Delta t$ exceeds the terminal relaxation time $\tau_{\infty}$ [as characterized using the $\mu_{1}(\Delta t)$ rescaling in Appendix B]. Please note that the determination of $\eta(\Delta t)$ using Eq. (3) for the liquid limit is notoriously difficult [20] due to the inaccuracy of $G(t) \approx 0$ for large times $t \rightarrow \Delta t$. Since the noisy $G(t)$ may even become negative, $\eta(\Delta t)$ can become nonmonotonic as may be seen for $T=0.5$. The observed maximum gives in this case a (very rough) guess of $\eta_{\infty}(T)$. The open symbols indicated in Fig. 13(b) have thus been obtained by terminating the integration of $G(t)$ as soon as a negative $G(t)$ fluctuation becomes too important.

Connection between $\mu(\Delta t)$ and $\eta(\Delta t)$. Since $\mu(\Delta t)$ and $\eta(\Delta t)$ are moments of the same function $G(t)$, they must be connected. It follows from Eq. (30) that

$$
\eta(\Delta t)=\frac{d}{d \Delta t}\left[\frac{\Delta t^{2}}{2} \mu(\Delta t)\right] .
$$

Since $\mu(\Delta t)=\mu_{\mathrm{sf}}(\Delta t), \eta(\Delta t)$ may be determined by numerical differentiation of the smooth $\mu_{\mathrm{sf}}(\Delta t)$ data. [In the liquid limit, $\mu_{\mathrm{sf}}(\Delta t)$ may be replaced by $\mu_{1}(\Delta t)$.] Using the same 

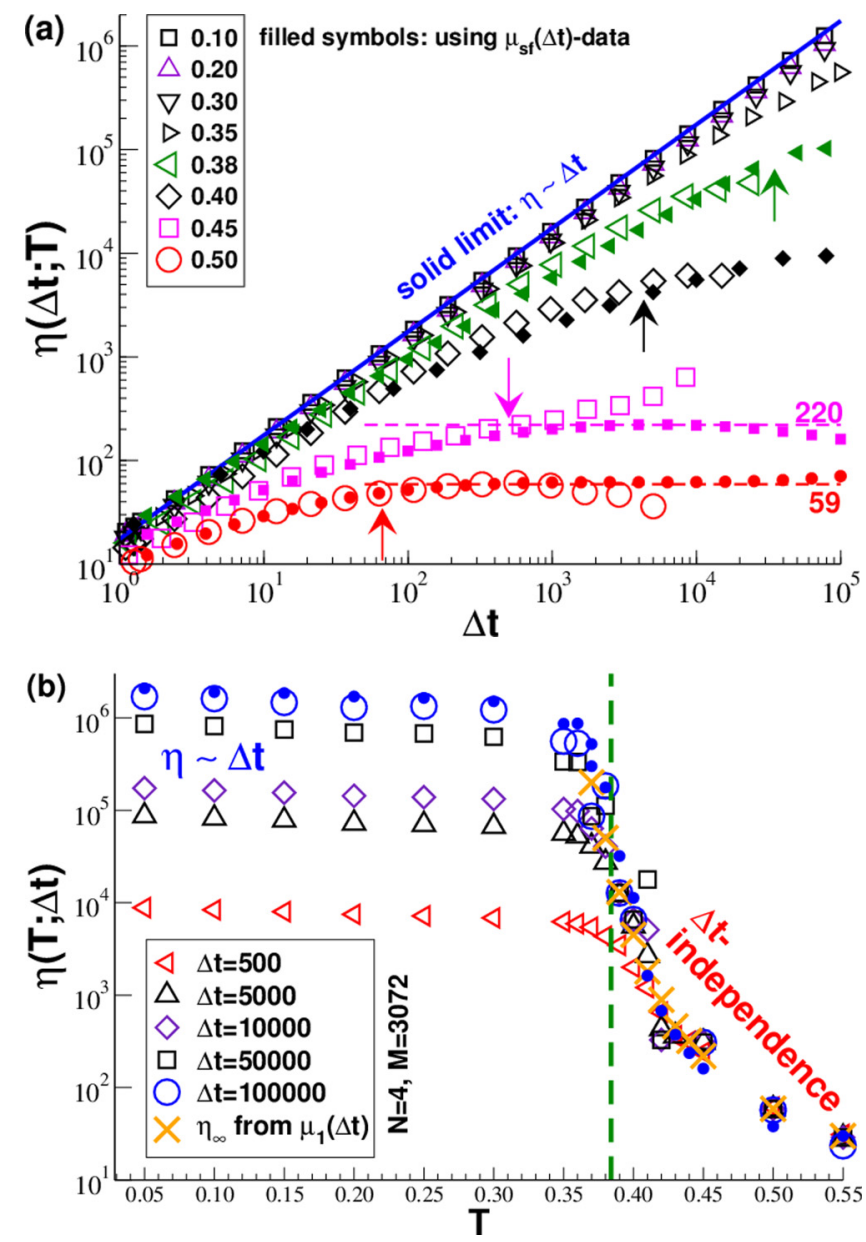

FIG. 13. Generalized shear viscosity $\eta$ with open symbols obtained using Eq. (3) and filled symbols using Eq. (37): (a) $\eta(\Delta t ; T)$ as a function of $\Delta t$ for several $T$. As shown by the solid line, $\eta(\Delta t)$ increases linearly in the solid limit. At higher temperatures $\eta(\Delta t)$ increases less strongly and eventually levels off. The two dashed lines indicate the limits $\eta(\Delta t) \rightarrow \eta_{\infty}(T)$ for $T=0.5$ and 0.45 . The vertical arrows mark for several temperatures the approximative position of the terminal relaxation time $\tau_{\infty}(T)$. (b) $\eta(T ; \Delta t)$ as a function of $T$ for different $\Delta t$. The crosses represent $\eta_{\infty}(T)$ from the inset of Fig. 17.

notations as in Eq. (32), this implies

$$
\eta(\Delta t)=e^{y(x)-x} y^{\prime}(x) .
$$

Results obtained for several temperatures are shown by the filled symbols in Fig. 13. While both methods yield equivalent results for small temperatures and for small $\Delta t$, the second method allows a slightly improved characterization of the plateau value $\eta_{\infty}$ in the liquid limit. Even more importantly, it follows directly from Eq. (36) that Eq. (2) must hold for $\Delta t \gg \tau_{\infty}$. Using in addition that $\mu_{\mathrm{sf}}(\Delta t) \approx \mu_{1}(\Delta t)$ in this limit it is readily seen that this is equivalent to the well-known Helfand-Einstein relation for the shear viscosity [20,35-37]. We have used Eq. (2) in Appendix B to determine $\eta_{\infty}(T)$ from $\mu_{1}(\Delta t)$. The corresponding data are indicated by the two horizontal dashed lines for $T=0.5$ and 0.45 in Fig. 13(a) and by crosses in Fig. 13(b). As one expects, it is seen that $\eta(\Delta t) \rightarrow \eta_{\infty}(T)$ in the large- $\Delta t$ limit.
Continuous behavior. As may be seen from Fig. 13(b), $\eta(T ; \Delta t)$ increases monotonically with decreasing temperature around $T_{\mathrm{g}}$ for all $\Delta t$. Albeit this increase becomes for larger $\Delta t$ more dramatic and numerically more difficult to describe, it remains continuous for all $\Delta t$, especially for the asymptotic limit $\eta_{\infty}(T)$. This is expected from the $\mu_{\text {sf }}$ data presented in Figs. 3 and 6. Interestingly, the last argument can be turned around. If one accepts that $\eta(\Delta t ; T)$ is monotonic and continuous for both $\Delta t$ and $T$, this implies that

$$
\mu_{\mathrm{sf}}(\Delta t ; T)=\mu(\Delta t ; T)=\frac{2}{\Delta t^{2}} \int_{0}^{\Delta t} d t \eta(t ; T)
$$

must have the same properties [49].

\section{E. Standard deviation $\delta G(t)$}

As we have already pointed out in Sec. VA, the statistics of $G(t)$ deteriorates for $t \rightarrow \Delta t$ since we have naturally used gliding averages along the time series [Eq. (12)] to compute this property. More importantly, it can be seen from Fig. 11 that fluctuations of $G(t)$ become stronger around $T_{\mathrm{g}}$. Both observations are confirmed quantitatively in Fig. 14 where we present the standard deviation $\delta G(t)$. Using doublelogarithmic coordinates $\delta G(t)$ is shown in the inset for several temperatures. It is seen that $\delta G(t)$ increases strongly for $t \rightarrow$ $\Delta t$. This effect is particularly pronounced for $T=0.35$. Using a similar representation as in Fig. 7 for $\delta \mu_{\mathrm{sf}}(T)$, the main panel of Fig. 14 presents $\delta G(T)$ as a function of temperature for several times $t$. As one expects, one observes a strong peak near $T_{\mathrm{g}}$. Interestingly, this peak is more pronounced as the one for $\delta \mu_{\mathrm{sf}}(T)$ and $\Delta t=10^{5}$. As we have shown in Sec. VB, $\mu_{\mathrm{sf}}(\Delta t)$ is given by the integral (1) over $G(t)$. It is natural to attempt similarly to describe the variance $\delta \mu_{\mathrm{sf}}^{2}(\Delta t)$ in terms of the variance $\delta G(t)^{2}$ using the weighted average

$$
\delta \mu_{\mathrm{sf}}^{2}(\Delta t) \approx \frac{2}{\Delta t^{2}} \int_{0}^{\Delta t} d t(\Delta t-t) \delta G(t)^{2} .
$$

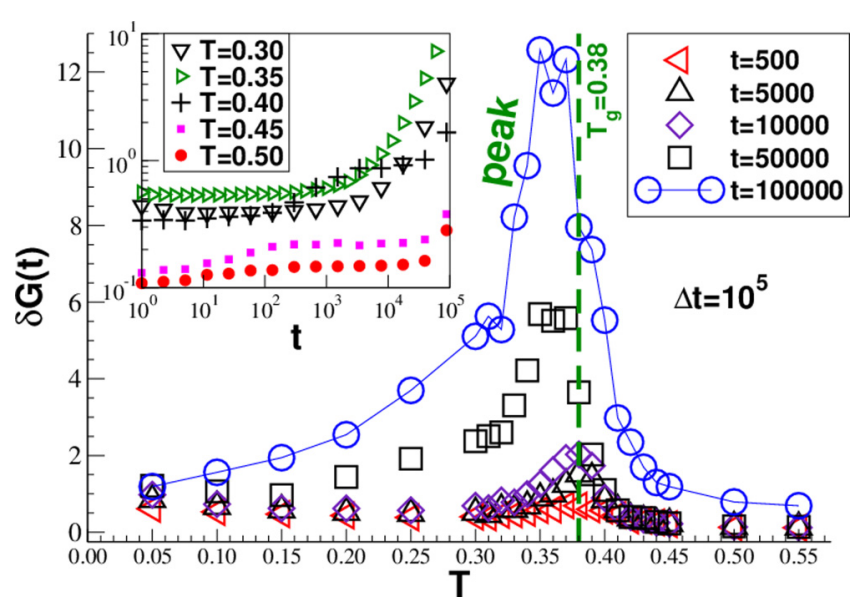

FIG. 14. Standard deviation $\delta G(t)$ of the relaxation modulus $G(t)$ presented in Fig. 11. All data have been obtained with gliding averages over time series of length $\Delta t=10^{5}$. Inset: double-logarithmic representation of $\delta G(t)$ vs time $t$ for several temperatures $T$. Main panel: $\delta G$ as a function of temperature for several times $t$ as indicated. $\delta G$ is nonmonotonic with a strong maximum slightly below $T_{\mathrm{g}}$. 

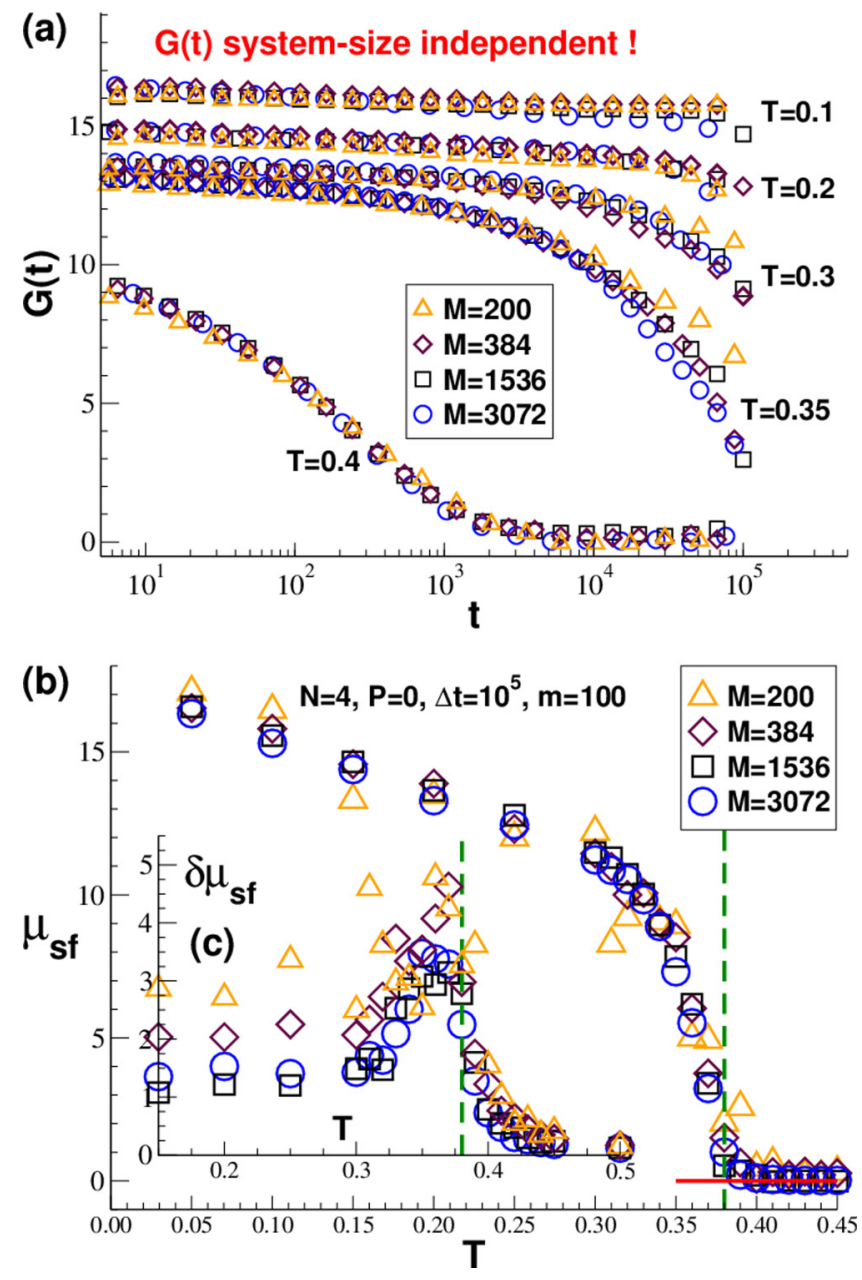

FIG. 15. Test of system-size effects using the four chain numbers $M=200,384,1536$, and 3072: (a) Shear-stress relaxation modulus $G(t)$ for five temperatures $T$. (b) $\mu_{\mathrm{sf}}\left(T ; \Delta t=10^{5}\right)$ for several $M$ demonstrating the expected system-size independence of the continuous transition of the shear modulus for finite sampling times $\Delta t$. (c) Standard deviation $\delta \mu_{\mathrm{sf}}(T)$ as a function of temperature $T$ for $\Delta t=10^{5}$.

This relation is a two-point approximation assuming that the standard deviations at different times are decorrelated. As shown by the filled symbols in Fig. 7, we have used Eq. (39) to predict $\delta \mu_{\mathrm{sf}}(\Delta t)$ using the data for $\delta G(t)$. The simple two-point approximation works quite reasonably, especially in the solid limit and the liquid limit. However, it overpredicts $\mu_{\mathrm{sf}}(\Delta t)$ around $T_{\mathrm{g}}$. [Note that a much better fit of the measured standard deviation $\delta \mu_{\text {sf }}$ is obtained if we replace in Eq. (39) the variances by the corresponding standard deviations. Unfortunately, this is more difficult to justify.] A closer inspection of higher order (three and four point) correlations is thus needed. This is beyond the scope of this paper. We only note that much longer time series are warranted to clarify this issue.

\section{SYSTEM-SIZE EFFECTS}

We comment finally on ongoing work on system-size effects presenting data obtained for four chain numbers $M=$ 200, 384, 1536, and 3072. The largest number $M=3072$, corresponding to a total mass $n=N M=12288$, is assumed everywhere else in the paper. As before, all data have been obtained for $N=4$ by averaging over $m=100$ configurations and three shear planes.

Figure 15(a) shows the shear-stress relaxation modulus $G(t)$ for five selected temperatures $T$. All data are obtained using the relation (20) for systems with quenched (sluggish) shear stresses [24]. As can be seen, $G(t)$ is essentially $M$ independent for all $T$. (Our smallest system with $M=200$ differs slightly for $T=0.3$ and 0.35 for long times. It relaxes more slowly. This observation is similar to the results reported in Ref. [50], but longer simulation runs are needed to scrutinize this effect.) Since $G(t)$ is $M$ independent and since the shear modulus $\mu_{\mathrm{sf}}$ obtained by Eq. (17) is equivalent to the integral $\mu(\Delta t)$ over $G(t)$ [Eq. (1)], one also expects $\mu_{\mathrm{sf}}(T)$ to be $M$ independent. As shown in Fig. 15(b) for $\Delta t=10^{5}$ this holds for all our data. This demonstrates that the continuous transition of $\mu_{\mathrm{sf}}(T ; \Delta t)$ observed for finite sampling times $\Delta t$ is not due to finite-size effects, as may happen for standard phase transitions [44], but is expected to hold for asymptotically large configurations. Naturally, the same $M$ independence follows also (not shown) for the generalized shear-stress viscosity $\eta(\Delta t)[\mathrm{Eq}$. (3)] and the relaxation time $\tau(\Delta t)$ [Eq. (E1)].

Focusing again on the sampling time $\Delta t=10^{5}$, the standard deviation $\delta \mu_{\mathrm{sf}}(T)$ of the shear modulus $\mu_{\mathrm{sf}}(T)$ is given in Fig. 15(c). The central point for the current study is that $\delta \mu_{\mathrm{sf}}(T)$ becomes system-size independent for temperatures $T$ around the glass transition temperature $T_{\mathrm{g}}$ and beyond, i.e., $\delta \mu_{\mathrm{sf}} / \mu_{\mathrm{sf}} \approx 1$ holds irrespective of the system size for $T \approx T_{\mathrm{g}}$. One expects from Procaccia et al. [16] and Wittmer et al. [17] that the standard deviations $\delta G(t)$ and $\delta \mu_{\text {sf }}$ should decrease with $M$ in the solid limit $\left(T \ll T_{\mathrm{g}}\right)$ where plastic rearrangements become rare and uncorrelated and, hence, irrelevant. This is qualitatively confirmed by our data, but larger configuration ensembles (with $m \gg 10^{2}$ ) and system sizes (with chain numbers $M \gg 10^{4}$ ) are warranted to verify this expectation more precisely.

\section{CONCLUSION}

\section{A. Summary}

We have investigated by means of MD simulations a coarsegrained model of polymer glasses (Sec. II). The linear shear mechanical response of this model has been characterized from the (ensemble-averaged) expectation values of the (timeaveraged) contributions to the stress-fluctuation relation $\mu_{\mathrm{sf}}=$ $\mu_{\mathrm{A}}-\mu_{\mathrm{F}}=\mu_{\mathrm{A}}-\mu_{0}+\mu_{1}$ for the shear modulus (Sec. III), their corresponding standard deviations and cross correlations (Sec. IV), and using the shear-stress relaxation modulus $G(t)$ and its in general $\Delta t$-dependent moments $\mu, \eta$ (Sec. V) and $\tau$ (Appendix E). The relaxation modulus has been directly determined by means of the recently proposed general fluctuation-dissipation relation (20), which can be also used for systems where $\mu_{\mathrm{A}}=\mu_{0}$ does not hold. We emphasize the following central results:

(i) Key result I: The liquid-solid transition characterized by the shear modulus $\mu_{\mathrm{sf}}(T)$ depends on the sampling time $\Delta t$ (Fig. 6). It is continuous with respect to the temperature $T$ for all $\Delta t$ becoming, however, sharper with increasing $\Delta t$ (Fig. 3). 
(ii) Strong quenched shear stresses arise naturally below the glass transition. $\mu_{0}$ and $\mu_{1}$ increase thus dramatically (Figs. 4 and 5).

(iii) Since time and ensemble averages commute for $\mu_{\mathrm{A}}$, $\mu_{0}$, and $G(t)$, their expectation values do not depend on the sampling time $\Delta t$ while all other properties studied here, especially all standard deviations, do in principle.

(iv) Together with the observation that $\mu_{0} \gg \mu_{\mathrm{A}}$ below $T_{\mathrm{g}}$, the $\Delta t$ independence of $\mu_{\mathrm{A}}$ and $\mu_{0}$ implies that our low- $T$ configurations are not compatible with the assumption that the finite- $\Delta t$ time series are randomly drawn from an equilibrium time evolution of a liquid (Sec. IIIC). This falling out of equilibrium is not an artifact of the particular preparation (quench) history of our configurations, but a central generic feature of the glass transition.

(v) Key result II: While the glass transition characterized by $\mu_{\text {sf }}$ becomes continuously sharper on average with increasing $\Delta t$, increasingly strong fluctuations $\delta \mu_{\mathrm{sf}}$ between different configurations underly the transition (Fig. 7). The broad and lopsided distribution $p\left(\bar{\mu}_{\mathrm{sf}}\right)$ below $T_{\mathrm{g}}$ makes the prediction of $\bar{\mu}_{\mathrm{sf}}$ for a single configuration elusive (Fig. 8). It is thus insufficient to discuss only the average shear modulus at the glass transition, fluctuations need also to be considered theoretically.

(vi) A clear-cut order parameter of the glass transition is given by the dimensionless correlation coefficient $r_{01}$ of the time-averaged moments $\bar{\mu}_{0}$ and $\bar{\mu}_{1}$ showing that the transition is logarithmically shifted to lower $T$ with increasing $\Delta t$ (Fig. 10).

(vii) Key result III: The observed $\Delta t$ dependence of $\mu_{\mathrm{sf}}$ (Figs. 3, 6, and 12) and its contributions $\mu_{1}$ (Fig. 17) and $\mu_{\mathrm{F}}$ can be traced back to the finite time (time-averaged) stress fluctuations need to explore the phase space. This effect is perfectly described by the weighted integral $\mu(\Delta t)$ over the shear-stress relaxation modulus $G(t)$ [Eq. (1)] shown to be identical to $\mu_{\mathrm{sf}}(\Delta t)$.

(viii) Albeit some aging must occur in our systems, this shows that this must happen on much larger time scales and that within the $\Delta t_{\max }$ window available, time-translational invariance holds to high accuracy for the macroscopic properties of interest here.

(ix) The stress-fluctuation formula $\mu_{\text {sf }}$ corresponds to an equilibrium storage modulus only if $G(t)$ becomes constant over a sufficiently large time window. This is of relevance in the solid limit well below $T_{\mathrm{g}}$ and in the liquid limit for $\Delta t \gg \tau_{\infty}$. In all other cases, one should see $\mu_{\text {sf }}$ as a "generalized shear modulus" or a useful smoothing function of $G(t)$ [Eq. (30)], which also contains information related to dissipative processes (Sec. VC).

(x) Since $G(t, T)$ is monotonic and continuous with respect to $T$ and $\Delta t$ (Fig. 11), this implies the same behavior for $\mu_{\mathrm{sf}}(\Delta t, T)=\mu(\Delta t, T), \eta(\Delta t, T)$, and $\tau(\Delta t, T)$ as seen in Figs. 3, 6, 13, and 20.

(xi) Since $\mu=\mu_{\mathrm{sf}}$ in general and $\mu_{1}=\mu_{\mathrm{sf}}$ for higher temperatures where $\mu_{\mathrm{A}}=\mu_{0}$, this allows to express $G(t)$, $\mu(\Delta t), \eta(\Delta t)$, and $\tau(\Delta t)$ above $T_{\mathrm{g}}$ in terms of the numerically better behaved $\mu_{1}(\Delta t, T)$ [Eqs. (32), (37), and (E3)]. Using these effectively low-pass filters, one avoids some of the problems related to the precision of the tail of $G(t)$. (xii) The $1 / \Delta t$ decay of $\mu_{\mathrm{sf}} \approx \mu_{1}$ at high temperatures [Eq. (2)] allows the determination of the shear viscosity $\eta_{\infty}$ (Fig. 17). This limit of $\mu_{\text {sf }}$ is equivalent to the well-known Helfand-Einstein relation for the shear viscosity.

(xiii) We have verified by varying the number of chains that the three key results stated in the Introduction are not due to system-size effects (Fig. 15).

\section{B. Outlook}

While $\mu_{\text {sf }}$ and its contributions $\mu_{\mathrm{A}}, \mu_{\mathrm{F}}, \mu_{0}$, and $\mu_{1}$ do not depend on the system size, this is more intricate for the corresponding standard deviations and must be addressed in the future following recent work on colloidal glasses [16] and on self-assembled networks [17]. The latter study suggests a strong self-averaging for the affine shear modulus $\mu_{\mathrm{A}}$, i.e., $\delta \mu_{\mathrm{A}} \sim 1 / M^{1 / 2}$, and a complete lack of self-averaging for $\mu_{0}$ and $\mu_{1}$, i.e., $\delta \mu_{0} \sim \delta \mu_{1} \sim M^{0}$, for all temperatures. As already seen in Fig. 15(c), we expect that further simulations with larger configurations will confirm that

$$
\delta G(t) \sim \delta \mu_{\mathrm{F}} \sim \delta \mu \sim \delta \eta \sim M^{0}
$$

around and above the glass transition (lack of self-averaging). In this limit, long-ranged viscoelastically interacting activated events should dominate the plastic reorganizations of the particle contacts [51]. At much lower temperatures, some self-averaging must become relevant, i.e., one expects

$$
\delta G(t) \sim \delta \mu_{\mathrm{sf}} \approx \delta \mu \sim 1 / M^{\alpha} \text { with } 0<\alpha \leqslant 1 / 2 .
$$

Our work on self-assembled transient networks [17] suggests strong self-averaging, i.e., $\alpha=\frac{1}{2}$, while the study by Procaccia et al. [16] points to a somewhat smaller exponent. Since the two temperature regimes must match, such a scaling would imply the existence of a characteristic length scale $\xi(T)$. Our guess is that such a length scale must be related to (and perhaps even be set by) the distance over which the sound waves generated by a given plastic particle rearrangement are able to trigger subsequent plastic events. Qualitatively different behavior is thus to be expected if three-dimensional polymer melts are compared to effectively two-dimensional melts confined to thin films.

We emphasize finally that albeit the presented work has focused on the shear-stress fluctuations, similar $\Delta t$-dependent results are expected for shear-strain fluctuations, mixed stressstrain fluctuations, and trajectory analysis in reciprocal space following Klix et al. [6,7]. It should be possible, e.g., to trace back the observed $\Delta t$ dependence in the latter case to a weighted integral over a wave-vector-dependent creep compliance.

\section{ACKNOWLEDGMENTS}

I.K. thanks the IRTG Soft Matter financed by the Deutsche Forschungsgemeinschaft (DFG) Grant No. GRK 1642/2 for financial support. We are indebted to A. N. Semenov (ICS, Strasbourg) and H. Xu (Univ. Lorraine, Metz) for helpful discussions. We thank the University of Strasbourg for CPU time through GENCI/EQUIP@MESO. 


\section{APPENDIX A: CANONICAL AFFINE SHEAR STRAINS}

Canonical affine transform. Let us consider a small shearstrain increment $\gamma$ in the $x y$ plane as it would be used to determine the shear relaxation modulus $G(t)$ by means of a direct out-of-equilibrium simulation [17,20,29-32]. For simplicity, all particles are in the principal simulation box [20]. It is assumed that all particle positions $\underline{r}$ and particle momenta $p$ follow the imposed "macroscopic" strain in a canonical affine manner according to [30]

$$
r_{x} \rightarrow r_{x}+\gamma r_{y} \text { and } p_{y} \rightarrow p_{y}-\gamma p_{x},
$$

where the negative sign in the second transform assures that Liouville's theorem [52] is satisfied.

General definitions. The (instantaneous) Hamiltonian $\hat{\mathcal{H}}$ of the given configuration will thus change as

$$
[\hat{\mathcal{H}}(\gamma)-\hat{\mathcal{H}}(\gamma=0)] / V \approx \hat{\tau}_{\mathrm{A}} \gamma+\frac{1}{2} \hat{\mu}_{\mathrm{A}} \gamma^{2} \text { for }|\gamma| \ll 1 \text {. }
$$

We thus define the instantaneous affine shear stress $\hat{\tau}_{\mathrm{A}}$ and the instantaneous affine shear modulus $\hat{\mu}_{\mathrm{A}}$ by

$$
\begin{gathered}
\hat{\tau}_{\mathrm{A}} \equiv \hat{\mathcal{H}}^{\prime}(\gamma) /\left.V\right|_{\gamma=0}, \\
\hat{\mu}_{\mathrm{A}} \equiv \hat{\mathcal{H}}^{\prime \prime}(\gamma) /\left.V\right|_{\gamma=0}=\left.\hat{\tau}_{\mathrm{A}}^{\prime}(\gamma)\right|_{\gamma=0},
\end{gathered}
$$

where a prime denotes a functional derivative with respect to the imposed canonical affine transformation [30]. It follows from the last equality in Eq. (A4) that

$$
\hat{G}(t=0)=\hat{\mu}_{\mathrm{A}}
$$

for the shear relaxation modulus of one configuration. Assuming the Hamiltonian $\hat{\mathcal{H}}=\hat{\mathcal{H}}^{\text {id }}+\hat{\mathcal{H}}^{\text {ex }}$ to be the sum of an ideal and an excess contribution $\hat{\mathcal{H}}^{\text {id }}$ and $\hat{\mathcal{H}}^{\text {ex }}$, similar relations apply for the corresponding contributions $\hat{\tau}_{\mathrm{A}}^{\mathrm{id}}$ and $\hat{\tau}_{\mathrm{A}}^{\mathrm{ex}}$ to $\hat{\tau}_{\mathrm{A}}=\hat{\tau}_{\mathrm{A}}^{\mathrm{id}}+\hat{\tau}_{\mathrm{A}}^{\mathrm{ex}}$ and for the contributions $\hat{\mu}_{\mathrm{A}}^{\mathrm{id}}$ and $\hat{\mu}_{\mathrm{A}}^{\mathrm{ex}}$ to $\hat{\mu}_{\mathrm{A}}=\hat{\mu}_{\mathrm{A}}^{\text {id }}+\hat{\mu}_{\mathrm{A}}^{\text {ex }}$. By explicitly applying Eq. (A1) to a given configuration using a broad range of shear strains $\gamma$, all four expansion coefficients $\hat{\tau}_{\mathrm{A}}^{\mathrm{id}}, \hat{\tau}_{\mathrm{A}}^{\mathrm{ex}}, \hat{\mu}_{\mathrm{A}}^{\mathrm{id}}$, and $\hat{\mu}_{\mathrm{A}}^{\mathrm{ex}}$ are in principle directly measurable observables irrespective of the specific Hamiltonian used [30].

Some useful formulas. As shown elsewhere [30], the ideal contributions become

$$
\begin{gathered}
\hat{\tau}_{\mathrm{A}}^{\mathrm{id}}=-\frac{1}{V} \sum_{i=1}^{n} p_{i, x} p_{i, y} / m_{i}, \\
\hat{\mu}_{\mathrm{A}}^{\mathrm{id}}=\frac{1}{V} \sum_{i=1}^{n} p_{i, x}^{2} / m_{i},
\end{gathered}
$$

where the sums run over all $n$ particles of mass $m_{i}$. Note that the minus sign for the ideal shear stress follows from the minus sign in Eq. (A1) required for a canonical transformation. Assuming a pairwise central conservative potential $\hat{\mathcal{H}}^{\mathrm{ex}}=\sum_{l} u\left(r_{l}\right)$ with $l$ labeling the interactions and $r_{l}$ the distance between the pair of monomers, one obtains the excess contributions [30]

$$
\begin{gathered}
\hat{\tau}_{\mathrm{A}}^{\mathrm{ex}}=\frac{1}{V} \sum_{l} r_{l} u^{\prime}\left(r_{l}\right) n_{l, x} n_{l, y}, \\
\hat{\mu}_{\mathrm{A}}^{\mathrm{ex}}=\frac{1}{V} \sum_{l}\left[r_{l}^{2} u^{\prime \prime}\left(r_{l}\right)-r_{l} u^{\prime}\left(r_{l}\right)\right] n_{l, x}^{2} n_{l, y}^{2}+\frac{1}{V} \sum_{l} r_{l} u^{\prime}\left(r_{l}\right) n_{l, y}^{2}
\end{gathered}
$$

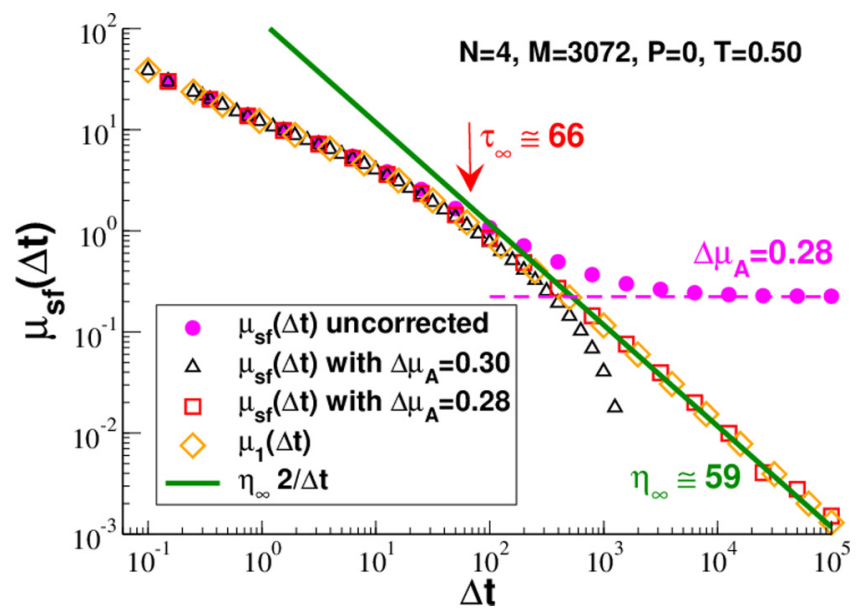

FIG. 16. $\mu_{\mathrm{sf}}(\Delta t)$ and its contribution $\mu_{1}(\Delta t)$ for the high temperature $T=0.5$ using logarithmic coordinates. The small filled circles represent uncorrected $\mu_{\mathrm{sf}}(\Delta t)$ data where we do not take into account the impulsive truncation corrections for $\mu_{\mathrm{A}}$ [43]. The uncorrected data saturate at a small, but finite value $\Delta \mu_{\mathrm{A}} \approx 0.28$ (dashed horizontal line). If correctly shifted (open squares), $\mu_{\mathrm{sf}}(\Delta t)=\mu_{1}(\Delta t)$ for all $\Delta t$ and $\mu_{\mathrm{sf}}(\Delta t)=\eta_{\infty} 2 / \Delta t$ for $\Delta t \gg \tau_{\infty}$ (solid line). The vertical arrow marks the terminal relaxation time $\tau_{\infty}=66$ for $T=0.5$ set as reference for the rescaling of $\mu_{1}(\Delta t)$ in Fig. 17.

with $\underline{n}_{l}=\underline{r}_{l} / r_{l}$ being the normalized distance vector. As one expects, Eq. (A8) is strictly identical to the corresponding offdiagonal term of the Irving-Kirkwood stress tensor [20]. The index " $A$ " for the shear stress has thus been dropped in other parts of this paper. The last term in Eq. (A9) takes into account the excess contribution of the average normal pressure [43].

Comments. Similar relations are obtained for the $x z$ and the $y z$ planes. For an isotropic system, the averages of all three affine shear moduli are finite and equal. We keep the index " $\mathrm{A}$ " to remind that the (time and ensemble averaged) $\mu_{\mathrm{A}}$ assumes a strictly affine strain without relaxation. It thus provides only an upper bound to the shear modulus of the configuration $[2,11,26-28,30]$. Please note that $\hat{\mu}_{\mathrm{A}}^{\mathrm{ex}}$ depends on the second derivative $u^{\prime \prime}(r)$ of the pair potential. As emphasized in Appendix B (Fig. 16), impulsive corrections need to be taken into account due to this term if the first derivative $u^{\prime}(r)$ of the potential is not continuous [43]. Unfortunately, this is the case at the cutoff of the LJ potential used in the current study (Sec. II A).

\section{APPENDIX B: HIGH-T LIMIT OF $\mu_{\mathrm{sf}}(\Delta t)$ AND $\mu_{1}(\Delta t)$}

Introduction. We address now three points, which, albeit valid in principle for all $T$, are in practice only relevant at sufficiently high temperatures where $\Delta t_{\max }=10^{5} \gg \tau_{\infty}(T)$. [An estimate of the terminal relaxation time $\tau_{\infty}(T)$ will be given below.] These points are illustrated for one temperature $(T=0.5)$ in Fig. 16 where we present $\mu_{\mathrm{sf}}(\Delta t)$ and $\mu_{1}(\Delta t)$ using double-logarithmic coordinates. This allows to pay attention to the large- $\Delta t$ behavior where both observables become very small. We remind that according to Eq. (25) one expects $\mu_{\mathrm{sf}}(\Delta t)=\mu_{1}(\Delta t)$ for all $\Delta t$ in the liquid limit where $\mu_{\mathrm{A}}=\mu_{0}$. 
Truncation effect. The first, more technical point we want to make concerns the truncation corrections due to the contribution of the second derivative of the potential to the affine shear modulus $\mu_{\mathrm{A}}$ discussed in Ref. [43]. As shown by the filled disks, the bare, uncorrected values of $\mu_{\mathrm{sf}}(\Delta t)$ saturate at some finite, positive value $\Delta \mu_{\mathrm{A}}$ as indicated by the dashed horizontal line. Albeit the effect is small, this finding is clearly unphysical since the true thermodynamic shear modulus of a liquid must rigorously vanish for large $\Delta t$ [48]. This is essentially the case if the data for the affine shear modulus are shifted, $\mu_{\mathrm{A}} \rightarrow \mu_{\mathrm{A}}-\Delta \mu_{\mathrm{A}}$, using the constant $\Delta \mu_{\mathrm{A}} \approx 0.3$ suggested by the histogram method described in Ref. [43]. Unfortunately, as can be seen from the open triangles, using this value for $\Delta \mu$, $\mu_{\mathrm{sf}}(\Delta t)$ is yet not identical to $\mu_{1}(\Delta t)$ for large $\Delta t$. Since it is currently not possible using the histogram method to obtain a more precise value for $\Delta \mu_{\mathrm{A}}$ [53], we have fine tuned $\Delta \mu_{\mathrm{A}}$ by insisting on $\mu_{\mathrm{sf}}(\Delta t) \approx \mu_{1}(\Delta t)$ for all $\Delta t$. This yields the refined shift constant $\Delta \mu_{\mathrm{A}} \approx 0.28$ used for the open squares. Similar values are obtained for other temperatures above $T_{\mathrm{g}}$. Using these more precise $\Delta \mu_{\mathrm{A}}$ values, one confirms the $1 / \Delta t$ asymptote (bold solid line) for $\mu_{\mathrm{sf}}(\Delta t)$ and $\Delta t \gg \tau_{\infty}$ expected on general grounds for finite-sampling-time corrections of time-preaveraged fluctuations [11,17,29-32,44].

Shear viscosity. This leads us to the second point we want to make. By fitting the amplitude of Eq. (2) given in the Introduction, one obtains in fact a rather precise estimate of the shear viscosity $\eta_{\infty}(T)$. This relation is a direct consequence of the key result Eq. (1) as shown in Sec. VD. Due to the tricky determination of the truncation shift constant $\Delta \mu_{\mathrm{A}}$, it is even more convenient to use directly $\mu_{1}(\Delta t)$ instead of $\mu_{\mathrm{sf}}(\Delta t)$ in the liquid limit as illustrated in Fig. 17(a). As shown by the thin dashed lines, it is thus possible to estimate $\eta_{\infty}(T)$ for temperatures down to $T \approx 0.38$ [54]. As seen from Fig. 17(b), the obtained values (crosses) are quite reasonable: $\eta_{\infty}(T)$ increases both monotonically and continuously over four orders of magnitude between $T=0.55$ and 0.38 . The data can be well fitted (bold line) using a Vogel-Fulcher-Tammann (VFT) law

$$
\eta_{\infty}(T) \approx 1.0 \exp [0.78 /(T-0.31)] \text { for } T \geqslant 0.37 \text {. }
$$

For slightly lower temperatures, Eq. (2) allows at least to guess the power-law amplitude. A possible value is indicated for $T=0.37$. By tracing $\mu_{1}(T ; \Delta t) \Delta t / 2$ for several $\Delta t$ (open symbols), one obtains an additional simple test of the observed values and lower bounds for $\eta_{\infty}(T)$ at even smaller temperatures. While a perfect data collapse is observed for large $T$ or $\Delta t$, the scaling naturally fails in the opposite limit.

Terminal relaxation time. Being the third point we want to make here, this failure allows an estimation of the terminal relaxation time $\tau_{\infty}(T)$ of the system and the sampling time $\Delta t \gg \tau_{\infty}(T)$ needed. For instance, $\Delta t=10000$ (diamonds) becomes insufficient for $T \approx 0.39$ and $\tau_{\infty}(T)$ should be of this order. This estimation of $\tau_{\infty}(T)$ can be made more quantitative by attempting a scaling plot for $\mu_{1}(\Delta t ; T)$ as shown in Fig. 17(c). We trace here the dimensionless $y=$ $\mu_{1}(\Delta t ; T) \tau_{\infty}(T) / \eta_{\infty}(T)$ as a function of the reduced sampling time $x=\Delta t / \tau_{\infty}(T)$ using the $\eta_{\infty}(T)$ values obtained from Eq. (2). The terminal relaxation times $\tau_{\infty}(T)$ are fixed by imposing a data collapse around $x \approx 1$ and by setting $\tau_{\infty}(T=0.5) \approx 66$ as a reference. This reference is indicated
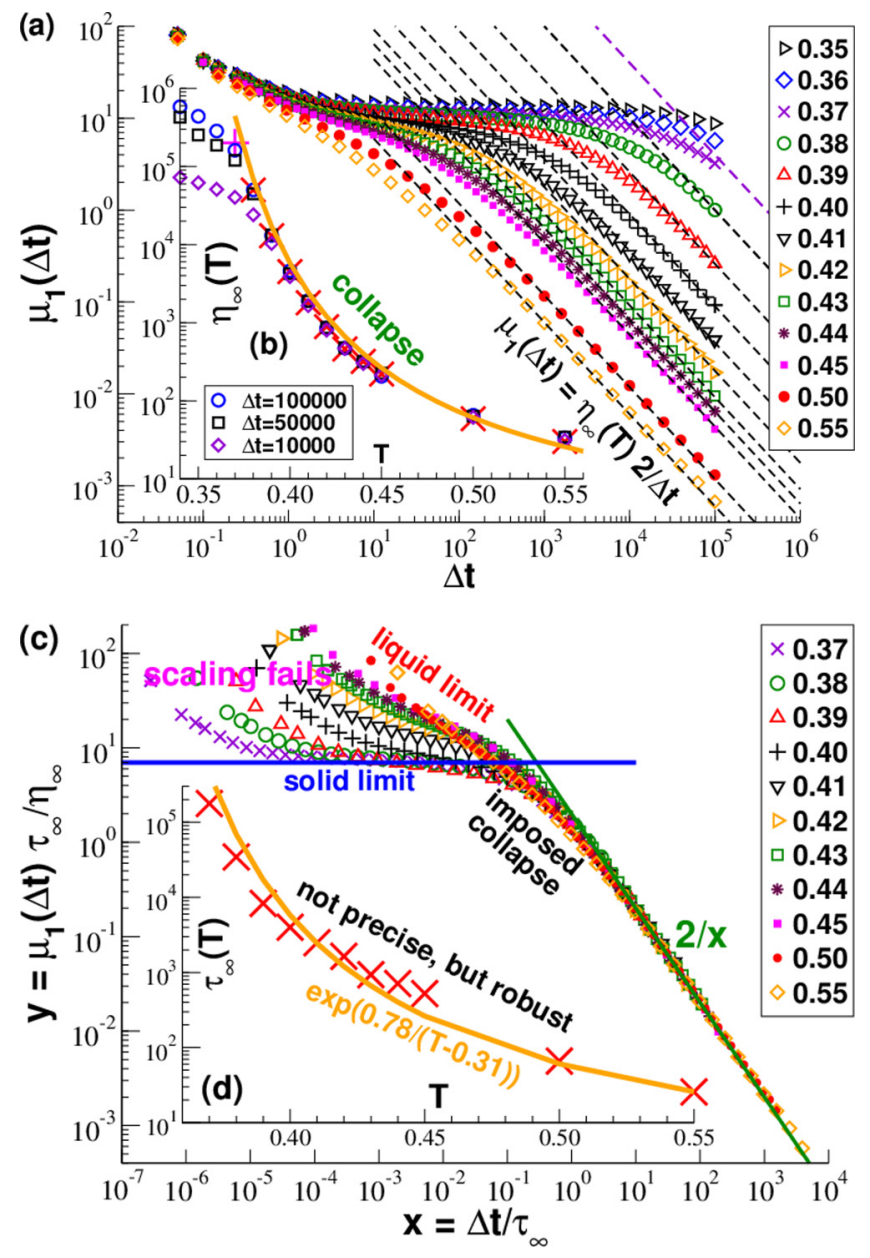

FIG. 17. $\mu_{1}(\Delta t)$ for higher temperatures where $\mu_{\mathrm{A}}=\mu_{0}$ holds: (a) Using double-logarithmic coordinates, the shear viscosity $\eta_{\infty}(T)$ can be estimated for $T \gtrsim 0.38$ by fitting $\mu_{1}(\Delta t)=\eta_{\infty} 2 / \Delta t$ as indicated by the dashed lines. (b) $\eta_{\infty}(T)$ increases over four orders of magnitude between $T=0.55$ and 0.38 . The plus is a fair guess for $T=0.37$. For comparison, we indicate $\mu_{1}(T ; \Delta t) \Delta t / 2$ for $\Delta t=$ 100000,50000 , and 10000 (open symbols). The bold line represents Eq. (B1). (c) Characterization of $\tau_{\infty}(T)$ by tracing $y=\mu_{1}(\Delta t) \tau_{\infty} / \eta_{\infty}$ vs $x=\Delta t / \tau_{\infty}$. (d) Terminal relaxation time $\tau_{\infty}(T)$ used for the rescaling of $\mu_{1}(\Delta t)$.

in Fig. 16 by a vertical arrow. It is motivated by the more systematic but numerically more difficult operational definition (E1) discussed in Appendix E. We thus obtain, e.g., $\tau_{\infty}(T=0.39) \approx 8300$ consistently with the failure of the scaling observed for the $\Delta t=10000$ data at $T=0.39$ in Fig. 17(c). The terminal relaxation times determined using the $\mu_{1}(\Delta t)$ rescaling are indicated in Fig. 17(d) (crosses). A dramatic monotonic increase over (again) four orders of magnitude is observed between $T=0.55$ and 0.37 . The bold line compares the $\tau_{\infty}$ data with the same VFT law Eq. (B1) used for the viscosity.

Summary. It is possible to obtain reasonable values for $\eta_{\infty}(T)$ and $\tau_{\infty}(T)$ from the asymptotic $1 / \Delta t$ decay and the scaling of $\mu_{1}(\Delta t ; T)$ for not too low temperatures. These values are given in Table II. Note that $\eta_{\infty}(T)$ has been determined with a much higher precision than $\tau_{\infty}(T)$. We emphasize that both $\eta_{\infty}(T)$ and $\tau_{\infty}(T)$ are completely continuous and no jump 
singularity is observed. Judging from the available data, it appears to be reasonable that by extending in the near future the production runs up to $\Delta t_{\max }=10^{6} \mathrm{using}$ the same system size or $\Delta t_{\max }=10^{7}$ using smaller systems, reliable values for both quantities down to $T=0.35$ are feasible.

\section{APPENDIX C: DETERMINATION OF $G(t)$}

Definitions and motivation. A central rheological property characterizing both liquids and solid elastic bodies is the shear relaxation modulus $G(t)[20,42,46,48]$. Assuming for simplicity an isotropic system, $G(t) \equiv \delta \tau(t) / \gamma$ may be obtained from the stress increment $\delta \tau(t)=\left\langle\hat{\tau}(t)-\hat{\tau}\left(0^{-}\right)\right\rangle$ after a small step strain with $|\gamma| \ll 1$ has been imposed at time $t=0$. A schematic representation of $G(t)$ is given in Fig. 18. The direct numerical computation of $G(t)$ by means of an out-of-equilibrium simulation, using the response to an imposed strain increment, is for technical reasons in general tedious [17,20,29-32]. It is thus of high importance to compute $G(t)$ correctly and efficiently "on the fly" by means of the appropriate linear-response fluctuation-dissipation relation for the standard canonical ensemble at imposed particle number $n$, volume $V$, shear strain $\gamma$, and temperature $T[20,42]$.

Shear-stress autocorrelation function. It is widely assumed $[6,20]$ that quite generally $G(t)=c(t)$ must hold with the shear-stress ACF $c(t)$ as defined in Eq. (18). A schematic representation of $c(t)$ is given in Fig. 18 and data for $c(t)$ as a function of temperature $T$ for several times $t$ in Fig. 19 . As indicated in Fig. 18, $c(t=0)=\mu_{0}$ with $\mu_{0}$ as defined by Eq. (14) in the main text. The opposite long-time limit $c(t=\Delta t)$ is slightly more intricate. It is of conceptional importance that for solid bodies, such as permanent elastic networks [11,29,30,32],

$$
c(t=\Delta t)=\mu_{1}=\left\langle\bar{\mu}_{1}\right\rangle \text { with } \bar{\mu}_{1} \equiv \beta V \overline{\hat{\tau}}^{2} .
$$

As shown in Appendix D, this relation may also be justified as the specific limit of a more general relation (D10) if plastic rearrangements can be neglected on the time scale probed. As shown in Fig. 19, Eq. (C1) holds quite generally

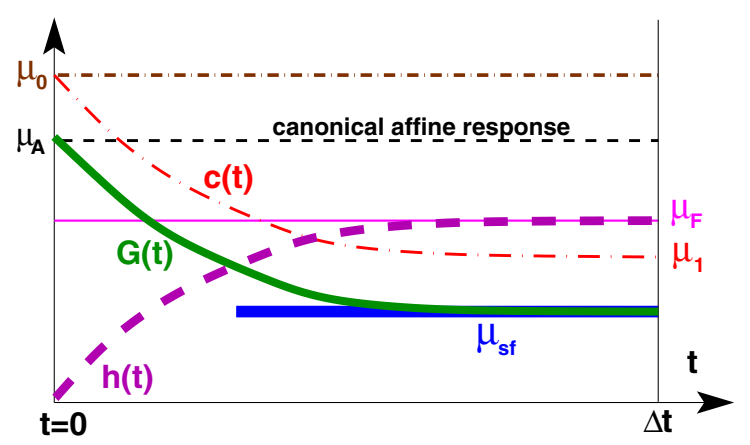

FIG. 18. Sketch of properties of interest for the determination of the shear-stress relaxation modulus $G(t)$ focusing on solids. Static properties are indicated by horizontal lines, $G(t)$ by the bold solid line, $c(t)$ by the thin dashed-dotted line, and $h(t)=c(0)-c(t)$ by the bold dashed line. A canonical affine shear transformation at $t=0$ implies $G(t=0)=\mu_{\mathrm{A}}$ while for large times $G(t) \rightarrow \mu_{\mathrm{sf}}$. At variance to this, $c(t)$ decays from $c(t=0)=\mu_{0}$ to $c(t=\Delta t)=\mu_{1}$. In general, $\mu_{\mathrm{A}} \neq \mu_{0}$, hence, $G(t) \neq c(t)$.

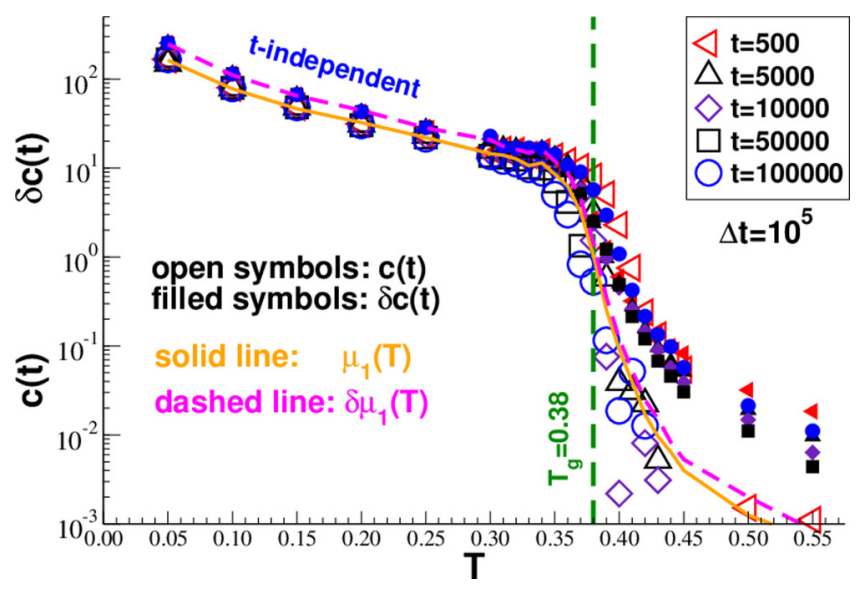

FIG. 19. Ensemble-averaged ACF $c(t)$ (open symbols) and associated standard deviation $\delta c(t)$ (filled symbols) as a function of temperature $T$ for several times $t$. Note that $\delta c(t)$ is always larger $c(t)$ for all $T$ and $t$. The bold solid line indicates $\mu_{1}(T)$ for $\Delta t=10^{5}$, the dashed line the corresponding standard deviation $\delta \mu_{1}(T)$. Below $T_{\mathrm{g}}$, one observes $c(t) \approx \mu_{1}(T)$ and $\delta c(t) \approx \delta \mu_{1}(T)$.

for temperatures below $T_{\mathrm{g}}$ and the fluctuations of the ACF are perfectly described by $\delta c(t) \approx \delta \mu_{1}(T)$ for a broad range of times $t$. As emphasized in Refs. [29,30], an important consequence of $c(t) \rightarrow \mu_{1}$ is now that $G(t)=c(t)$ is inconsistent with $G(t) \rightarrow \mu_{\mathrm{sf}}$ and the stress-fluctuation formula $\mu_{\mathrm{sf}}=\left(\mu_{\mathrm{A}}-\mu_{0}\right)+\mu_{1}$ which must hold quite generally for solid bodies [2,11,25-28,30].

More general relation. This problem is resolved by means of the slightly more general fluctuation-dissipation relation $[12,17,24,31,32]$ stated by Eqs. (12) and (20) in the main text. This relation has been called a "simple-average expression" in [31,32] since both terms $\bar{\mu}_{\mathrm{A}}$ and $\bar{h}(t)$ transform as simple averages [20] between the conjugated ensembles at constant shear strain and constant shear stress. For elastic solids, this is one possibility to derive Eq. (12) within a few lines [31]. Please note that the ACF $c(t)$ and the MSD $h(t)$ are related by [46]

$$
h(t)=c(0)-c(t)=\mu_{0}-c(t)
$$

Using Eq. (C1) and $h(t) \rightarrow \mu_{\mathrm{F}}$ for large times, this implies that Eq. (20) is consistent with

$$
\lim _{t \rightarrow \Delta t} G(t)=\mu_{\mathrm{sf}}(\Delta t)=\mu_{\mathrm{A}}-\mu_{\mathrm{F}}(\Delta t)
$$

as it should. As shown in Ref. [24], Eq. (20) remains statistically well behaved below $T_{\mathrm{g}}$ despite the strong fluctuations of the frozen shear stresses in the different shear planes since the frozen stresses drop out of the stress difference computed for $h(t)$. See Sec. VE for the low-temperature behavior of the standard deviation $\delta G(t)$. We emphasize finally that since $\mu_{\mathrm{A}}=\mu_{0}$ holds in the liquid limit (Sec. III C), Eq. (20) simplifies to $G(t)=c(t)$ as one expects.

\section{APPENDIX D: RELATION BETWEEN $G(t)$ AND $\mu_{\mathrm{sf}}(\Delta t)$}

Some useful properties of a functional. With $y(t)$ being an arbitrary well-behaved function of $t$, let us consider the linear 
functional

$$
\mathcal{P}_{\Delta t}[y(t)] \equiv \frac{2}{\Delta t^{2}} \int_{0}^{\Delta t} d t(\Delta t-t) y(t)
$$

Interestingly, for a constant function

$$
y(t)=c_{0} \quad \text { we have } \mathcal{P}_{\Delta t}\left[c_{0}\right]=c_{0},
$$

i.e., the $\Delta t$ dependence drops out. This even holds to leading order if $y(t) \approx c_{0}$ only for large $t$ or for a finite $t$ window if this window becomes sufficiently large. Note that contributions at the lower boundary of the integral have a strong weight due to the factor $(\Delta t-t)$. If $y(t)$ is a strictly monotonically decreasing function, we have

$$
y(t=\Delta t)<\mathcal{P}_{\Delta t}[y(t)] .
$$

This inequality also holds if $y(t)$ is only in a finite, but broad, intermediate time window a monotonically decreasing function.

Time-translational invariance. Let us consider a time series $\left(x_{1}, \ldots, x_{n}, \ldots x_{N}\right)$ with entries $x_{n}$ sampled at equidistant time intervals $d t$. The time-averaged variance of this time series may be rewritten as

$$
\begin{aligned}
\overline{x^{2}}-\bar{x}^{2} & =\overline{\left(x_{n}-\bar{x}\right)^{2}}=\frac{1}{2 N^{2}} \sum_{n, m=1}\left(x_{n}-x_{m}\right)^{2} \\
& =\frac{2}{N^{2}} \sum_{s=0}^{N-1}(N-s) \bar{h}(s, N) .
\end{aligned}
$$

We have defined here the gliding average

$$
\bar{h}(s, N) \equiv \frac{1}{2} \frac{1}{N-s} \sum_{n=1}^{N-s}\left(x_{n+s}-x_{n}\right)^{2}
$$

which depends a priori on both $s$ and $N$. The latter representation is useful if the time series is stationary, i.e., timetranslational invariance can be assumed on average. Taking the expectation value $\langle\ldots\rangle$ over an ensemble of such time series yields

$$
\begin{gathered}
\left\langle\overline{x^{2}}-\bar{x}^{2}\right\rangle=\frac{2}{N^{2}} \sum_{s=0}^{N-1}(N-s) h(s) \\
\text { with } h(s) \equiv\langle\bar{h}(s, N)\rangle=\frac{1}{2}\left\langle\overline{\left(x_{s}-x_{0}\right)^{2}}\right\rangle
\end{gathered}
$$

being now a proper MSD depending only on the time increment as the one introduced in Eq. (19). In the continuum limit for $N \gg 1$ the latter result becomes

$$
\left\langle\overline{x^{2}}-\bar{x}^{2}\right\rangle=\mathcal{P}_{\Delta t}[h(t)],
$$

where we use that the time series has been sampled with equidistant time steps, i.e., $t \approx s d t$ and $\Delta t \approx N d t$.

Back to current problem. Setting $x(t) \equiv \sqrt{\beta V} \hat{\tau}(t)$ and assuming time-translational invariance for the sampled instantaneous shear stresses $\hat{\tau}$, Eq. (D8) thus implies

$$
\mu_{\mathrm{F}}(\Delta t) \equiv \mu_{0}-\mu_{1}(\Delta t)=\mathcal{P}_{\Delta t}[h(t)]
$$

for the $\Delta t$ dependence of the shear-stress fluctuations in agreement with the more direct demonstration given in Ref. [29].
Since $\mu_{0}$ does not depend explicitly on $\Delta t$, it follows using Eq. (C2) that

$$
\mu_{1}(\Delta t)=\mu_{0}-\mathcal{P}_{\Delta t}[h(t)]=\mathcal{P}_{\Delta t}[c(t)] .
$$

Importantly, this reduces to the relation $c(t=\Delta t)=\mu_{1}$ for solids if the ACF $c(t)$ becomes constant for large times. We have thus obtained a generalization of Eq. (15) being also valid for general viscoelastic fluids. Since $\mu_{\mathrm{A}}$ is constant, Eqs. (D2) and (20) imply

$$
\begin{aligned}
\mu_{\mathrm{sf}}(\Delta t) & \equiv \mu_{\mathrm{A}}-\mu_{\mathrm{F}}(\Delta t)=\mathcal{P}_{\Delta t}[G(t)] \\
& =\frac{2}{\Delta t^{2}} \int_{0}^{\Delta t}(\Delta t-t) G(t) d t
\end{aligned}
$$

in agreement with Eq. (1) stated in the Introduction.

\section{APPENDIX E: RELAXATION TIME $\tau(\Delta t)$}

It is tempting to consider as an additional moment over $G(t)$ the generalized $\Delta t$-dependent shear-stress relaxation time

$$
\tau(\Delta t) \equiv \frac{1}{\eta(\Delta t)} \int_{t=0}^{\Delta t} d t t G(t)
$$

with $\tau_{\infty} \equiv \lim _{\Delta t \rightarrow \infty} \tau(\Delta t)$ being the experimentally relevant terminal relaxation time of the system. Note that $\tau(\Delta t)=$ $\tau_{\infty}$ for all $\Delta t$ for a Maxwell fluid with $G(t) \sim \exp \left(-t / \tau_{\infty}\right)$ as observed, e.g., for equilibrium polymers [55], vitrimers [18,19], or self-assembled transient networks [17]. Unfortunately, Eq. (E1) must be dominated even more strongly by the upper bound of the integral over $G(t)$ than the generalized shear viscosity $\eta(\Delta t)$. Since $G(t)$ is strongly fluctuating for $t \rightarrow \Delta t_{\max }=10^{5}$, especially around $T_{\mathrm{g}}$ (Fig. 11), this leads to an unreliable estimation of $\tau(\Delta t)$ for $\Delta t \rightarrow \Delta t_{\max }$. This suggests to reexpress Eq. (E1) in terms of $\mu_{\mathrm{sf}}(\Delta t)$ with $\Delta t \leqslant$ $\Delta t_{\max }$. As seen from Eqs. (1) and (E1), the three moments $\mu(\Delta t), \eta(\Delta t)$, and $\tau(\Delta t)$ are related by

$$
\tau(\Delta t)=\Delta t-\left[\mu(\Delta t) \Delta t^{2} / 2\right] / \eta(\Delta t)
$$

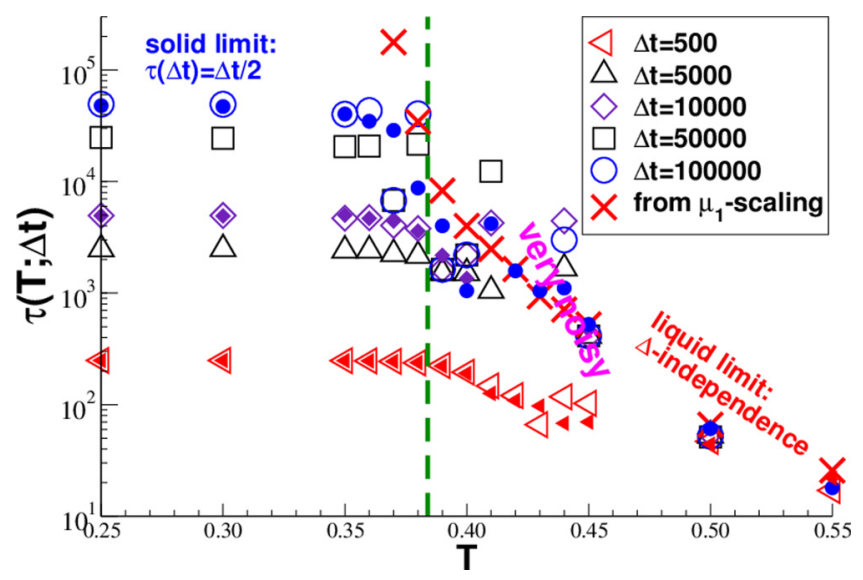

FIG. 20. Generalized shear-stress relaxation time $\tau(T ; \Delta t)$ for several $\Delta t$ with open symbols obtained using Eq. (E1) and filled symbols using Eq. (E3) taking advantage of the $\mu_{\mathrm{sf}}(\Delta t)$ data. For both methods, the data are noisy and unreliable around $T_{\mathrm{g}}$. This is at variance to the smooth $\tau_{\infty}(T)$ values (crosses) obtained in Fig. 17 from $\mu_{1}(\Delta t)$. 
One may thus obtain the generalized relaxation time from

$$
\tau(\Delta t)=e^{x}\left[1-1 / y^{\prime}(x)\right]
$$

with again $x \equiv \ln (\Delta t)$ and $y \equiv \ln \left(\mu_{\mathrm{sf}}(\Delta t) \Delta t^{2} / 2\right)$ fitted to sixth order and $\mu_{1}(\Delta t)$ replacing $\mu_{\mathrm{sf}}(\Delta t)$ for large temperatures.

Figure 20 presents $\tau(T ; \Delta t)$ as a function of temperature using half-logarithmic coordinates. The open symbols indicate values obtained using the direct integral (E1), filled symbols data using Eq. (E3), and the crosses the terminal relaxation times estimated using the rescaling of $\mu_{1}(\Delta t)$ presented in Fig. 17(c). The first two methods yield identical results for small $\Delta t$ and in the solid limit. As for $\eta(\Delta t)$, one observes that
$\tau(\Delta t)$ increases linearly in the solid limit where $\tau(\Delta t)=\Delta t / 2$ for $\Delta t \ll \tau_{\infty}(T)$. For the more interesting higher temperatures, the first method yields slightly erratic results although the integration is terminated at the first occurrence of a strong negative $G(t)$ fluctuation. The observed $\Delta t$ independence for large temperatures is thus trivially imposed and not the confirmation of an expected result. Unfortunately, similar rather erratic data are obtained using Eq. (E3) as shown for $\Delta t=10^{5}$. However, albeit very noisy, both data sets approach with increasing $\Delta t$ the terminal relaxation time estimated using the $\mu_{1}(\Delta t)$ rescaling (crosses). Being certainly not very precise (not even on the used logarithmic scale), the $\tau(\Delta t ; T)$ data are thus at least consistent with the $\mu_{1}(\Delta t)$ data.
[1] J.-L. Barrat, J.-N. Roux, J.-P. Hansen, and M. L. Klein, Europhys. Lett. 7, 707 (1988).

[2] D. Li, H. Xu, and J. P. Wittmer, J. Phys.: Condens. Matter 28, 045101 (2016).

[3] W. Götze, Complex Dynamics of Glass-Forming Liquids: A Mode-Coupling Theory (Oxford University Press, Oxford, 2009).

[4] G. Szamel and E. Flenner, Phys. Rev. Lett. 107, 105505 (2011).

[5] M. Ozawa, T. Kuroiwa, A. Ikeda, and K. Miyazaki, Phys. Rev. Lett. 109, 205701 (2012).

[6] C. L. Klix, F. Ebert, F. Weysser, M. Fuchs, G. Maret, and P. Keim, Phys. Rev. Lett. 109, 178301 (2012).

[7] C. L. Klix, G. Maret, and P. Keim, Phys. Rev. X 5, 041033 (2015).

[8] H. Yoshino and F. Zamponi, Phys. Rev. E 90, 022302 (2014).

[9] H. Yoshino, J. Chem. Phys. 136, 214108 (2012).

[10] A. Zaccone and E. M. Terentjev, Phys. Rev. Lett. 110, 178002 (2013).

[11] J. P. Wittmer, H. Xu, P. Polińska, F. Weysser, and J. Baschnagel, J. Chem. Phys. 138, 12A533 (2013).

[12] I. Kriuchevskyi, J. P. Wittmer, H. Meyer, and J. Baschnagel, Phys. Rev. Lett. 119, 147802 (2017).

[13] A. Andreanov, G. Biroli, and J.-P. Bouchaud, Eur. Phys. Lett. 88, 16001 (2009).

[14] P. Charbonneau, J. Kurchan, G. Parisi, P. Urbani, and F. Zamponi, Nat. Commun. 5, 3725 (2014).

[15] G. Biroli and P. Urbani, Nat. Phys. 12, 1130 (2016).

[16] I. Procaccia, C. Rainone, C. A. B. Z. Shor, and M. Singh, Phys. Rev. E 93, 063003 (2016).

[17] J. P. Wittmer, I. Kriuchevskyi, A. Cavallo, H. Xu, and J. Baschnagel, Phys. Rev. E 93, 062611 (2016).

[18] D. Montarnal, M. Capelot, F. Tournilhac, and L. Leibler, Science 334, 965 (2011).

[19] F. Smallenburg, L. Leibler, and F. Sciortino, Phys. Rev. Lett. 111, 188002 (2013).

[20] M. Allen and D. Tildesley, Computer Simulation of Liquids (Oxford University Press, Oxford, 1994).

[21] S. J. Plimpton, J. Comput. Phys. 117, 1 (1995).

[22] B. Schnell, H. Meyer, C. Fond, J. P. Wittmer, and J. Baschnagel, Eur. Phys. J. E 34, 97 (2011).

[23] J. Baschnagel, I. Kriuchevskyi, J. Helfferich, C. Ruscher, H. Meyer, O. Benzerara, J. Farago, and J. Wittmer, in Polymer Glasses, edited by C. Roth (Taylor \& Francis, London, 2016), p. 153.
[24] I. Kriuchevskyi, J. Wittmer, O. Benzerara, H. Meyer, and J. Baschnagel, Eur. Phys. J. E 40, 43 (2017).

[25] D. R. Squire, A. C. Holt, and W. G. Hoover, Physica (Amsterdam) 42, 388 (1969).

[26] J. F. Lutsko, J. Appl. Phys 64, 1152 (1988).

[27] J. P. Wittmer, A. Tanguy, J.-L. Barrat, and L. Lewis, Europhys. Lett. 57, 423 (2002).

[28] J.-L. Barrat, in Computer Simulations in Condensed Matter Systems: From Materials to Chemical Biology, Vol. 2, edited by M. Ferrario, G. Ciccotti, and K. Binder (Springer, Berlin, 2006), Vol. 704, pp. 287-307.

[29] J. P. Wittmer, H. Xu, and J. Baschnagel, Phys. Rev. E 91, 022107 (2015).

[30] J. P. Wittmer, H. Xu, O. Benzerara, and J. Baschnagel, Mol. Phys. 113, 2881 (2015).

[31] J. P. Wittmer, I. Kriuchevskyi, J. Baschnagel, and H. Xu, Eur. Phys. J. B 88, 242 (2015).

[32] J. P. Wittmer, H. Xu, and J. Baschnagel, Phys. Rev. E 93, 012103 (2016).

[33] The ensemble-averaged prediction is thus irrelevant for a single configuration. This does, however, not imply that a single configuration may not have a finite well-defined shear modulus.

[34] S. Alexander, Phys. Rep. 296, 65 (1998).

[35] J. Boon and S. Yip, Molecular Hydrodynamics (Dover, New York, 1980).

[36] M. P. Allen, D. Brown, and A. J. Masters, Phys. Rev. E 49, 2488 (1994).

[37] M. Allen, Phys. Rev. E 50, 3277 (1994).

[38] S. Frey, F. Weysser, H. Meyer, J. Farago, M. Fuchs, and J. Baschnagel, Eur. Phys. J. E 38, 11 (2015).

[39] In addition to the presented data, we have sampled systematically a broad range of chain lengths $N$ and cooling rates $\Gamma$. As one expects [22,38,23], the glass transition temperature $T_{\mathrm{g}}$ is found to depend somewhat on both parameters without changing the qualitative behavior.

[40] The second half of the tempering step at constant $P$ is used to determine the average volume $\bar{V}$ which is then used for the subsequent constant volume simulations.

[41] It would have theoretically been more exact to use here instead the fixed volume $\bar{V}$ of each configuration. But, since the volume fluctuations within the ensemble are tiny, this difference is numerically irrelevant. 
[42] J. Hansen and I. McDonald, Theory of Simple Liquids, 3rd ed. (Academic, New York, 2006).

[43] H. Xu, J. P. Wittmer, P. Polińska, and J. Baschnagel, Phys. Rev. E 86, 046705 (2012).

[44] D. P. Landau and K. Binder, A Guide to Monte Carlo Simulations in Statistical Physics (Cambridge University Press, Cambridge, 2000).

[45] This is, e.g., the case for self-assembled transient networks formed by reversibly bridging and breaking bonds between colloids [17]. In this case, $\mu_{\mathrm{A}}=\mu_{0}$ holds not only in the liquid regime, but also through the glass transition into the quenched amorphous solid limit.

[46] M. Doi and S. F. Edwards, The Theory of Polymer Dynamics (Clarendon, Oxford, 1986).

[47] T. Witten and P. A. Pincus, Structured Fluids: Polymers, Colloids, Surfactants (Oxford University Press, Oxford, 2004).

[48] M. Rubinstein and R. Colby, Polymer Physics (Oxford University Press, Oxford, 2003).

[49] Equation (38) may be obtained by integration by parts from Eq. (1) or by integration of Eq. (36). The integration constant vanishes as can be seen from the fact that both sides of Eq. (38) become $\mu_{\mathrm{A}}$ for $\Delta t \rightarrow 0$.

[50] L. Berthier, G. Biroli, D. Coslovich, W. Kob, and C. Toninelli, Phys. Rev. E 86, 031502 (2012).

[51] E. E. Ferrero, K. Martens, and J.-L. Barrat, Phys. Rev. Lett. 113, 248301 (2014).

[52] H. Goldstein, J. Safko, and C. Poole, Classical Mechanics, 3rd ed. (Pearson, London, 2001).

[53] Surprisingly, $\Delta \mu_{\mathrm{A}}$ depends somewhat on the time increment $\delta t_{\mathrm{MD}}$ of the velocity-Verlet integration step. A complete understanding and description of this technical issue is still missing. This observation is presumably due to small detailed balance violations of the MD integration scheme which lead to inconsistencies with the rigorous thermodynamic relations put forward in Ref. [43].

[54] $\eta_{\infty}(T)$ is most readily determined from the plateau at large $\Delta t$ of $\mu_{1}(\Delta t) \Delta t / 2$ traced using a half-logarithmic representation.

[55] M. Cates and S. Candau, J. Phys.: Condens. Matter 2, 6869 (1990). 